\author{
Proposal for Upgrading \\ the \\ Collider Detector at Fermilab
}

\title{
CDF Collaboration*
}

With the successful test run of CDF during September-Dctober, 1985 and the beginning of physics running later this year, now is the appropriate time to begin a specific program of detector upgrades so that the full physics potential of CDF at the Tevatron Collider can be exploited in an optimum way. We seek PAC guidance and approval of our plans to go beyond the original scope of CDF.

The basic design and physics goals of CDF have not changed substantially from our 1981 Design Report. Experience at the CERN Sp̄ps collider confirms that CDF will be a very powerful instrument for exploring new physics. Essentially all of the hardware will be completed for the first physics run in late 1886; final quantities of some front-end electronics and partial sampling in the forward hadron calorimeters had to be delayed until FY1887 because of actual funding schedules. Thus, by the beginning of 1987, we will have completed the base detector and will begin to explore the new physics regime made possible by the Tevatron.

Numerous suggestions have been made for enhancing the physics potential of CDF by building additional detector components. The collaboration has examined these in workshops and informal

discussions and is prepared to move forward with certain upgrades as soon as support can be secured. Because of our commitment to an aggressive and intensive physics program with the base detector, we have limited current upgrade plans to projects with very high priority. These high priority improvements are:

1) Completion of the Level-3 trigger system. This is a fully programmable trigger system based on a farm of ACP microprocessors that can run detailed analysis codes for event selection to reduce the rate of events logged for offline analysis. Level-3 will also be used to aid in monitoring the experiment and to isolate rare events for rapid analysis.

21) Construction of muon spectrometers (super-toroids) to provide muon identification, momentum measurements, and triggers in the intermediate angle range between the forward muon toroids and the central muon detectors where there is no muon coverage in the base plan.

*See Appendix A 
3) Construction of a transition radiation detector system to cover the forward/backward region for improving electron identification in this region where magnetic analysis is ineffective.

4) Development of a micro-vertex detector system to aid in identifying particles with intermediate lifetimes such as tau leptons and hadrons containing bottom quarks.

Each of these high priority upgrades differs in its stage of development and overall effort required. Therefore, we are treating them separately and we seek different kinds of advice and approval from the PAC. Nevertheless, all of these upgrades are very important to CDF and all must be completed in a timely fashion to best exploit the physics opportunities of Tevatron I.

It has long been recognized that a "FORTRAN-Programmable", Level-3 trigger system is very important when the collider luminosity exceeds roughly $10^{29} \mathrm{~cm}^{-2} \mathrm{~s}^{-1}$. Since this is likely to happen by the end of 1987, CDF decided to begin constructing a Level-3 system even though full funding has not been secured. After an extensive internal review, CDF decided to base its Level-3 system on the Fermilab ACP system. The system is described and its current status is summarized in Appendix B.

Funds to build the necessary data acquisition system interfaces and first pieces of the ACP farm were taken from contingency and were provided through the US/Japan Accord. However, new sources of support are required to complete Level-3 and bring it up to full performance. As indicated in Appendix B, approximately $\$ 367 \mathrm{~K}$ is needed for this. Ne are requesting PAC concurrence with our plans for Level-3 and its support for our efforts to secure funding to complete Level-3.

With the experience gained in assemblying CDF and moving large detector pieces in and out of the BO collision hall, it is now possible to design a single muon toroid system that covers the polar angle range $16^{\circ}$ to $55^{\circ}$. This system, the Muon Supertoroids, can thus join our existing forward/backward muon toroids and the central muon system giving continuous muon detection, triggering, and momentum determination over nearly 8 units of rapidity. The proposed muon upgrade is described in Appendix C. Final engineering of this system can be completed during FY87 and construction could be finished in FY89. Te are requesting PAC support of this upgrade so that final engineering and construction can begin as soon as possible.

In the forward/backward regions, electron identification can be improved significantly by adding a TRD system as described in Appendix D. Because the momentum analysis from the solenoid is essentially nonexistent at these small angles, we view the TRD system as necessary to bring our forward electron capabilities up to a level comparable with the central and end plug regions. This uniformity of response over the full acceptance is a very 
important part of the CDF design. The success of the LeingradFermilab TRD system in recent hyperon fixed target studies gives us confidence that ouch TRD systems can be engineered reliably. In the case of CDF, this is a natural addition because of the relatively long available free space between the end plug and the forward EM shower counters. Our program for the TRD system is to begin detailed engineering, design and prototyping studies early in FY87 so that construction of the chamber and radiator assemblies could begin by the end of that year, pending the successful outcome of the design effort. The entire system would be installed in CDF by the end of FY88. Therefore, we are seeking PAC support for the TRD upgrade to begin as soon as possible so that it can be completed before the end of FY 1988.

The power of micro vertex detectors has been demonstrated in a number of experiments. Indeed, this may be the only technique possible for identifying b-quark mesons and the tau leptons with sufficient reliability and efficiency to allow physics studies with these particles. CDF is engaged in a development project with the goal of installing a micro vertex detector based on Si strip technology after the first or second full running period (during FY88, most likely). The detector and the current R\&D activity is described in Appendix $\mathrm{E}$. We have reviewed the development project carefully within CDF and recognize that several milestones must be met before a full system can be built and installed. These include: 1) the successful operation of the special front-end electronics in conjunction with the silicon strips, 2) a complete mechanical design that maintains alignment of the Si strips without causing unacceptable backgrounds in other parts of CDF, and 3) physics studies based on actual CDF events which demonstrate that such secondary vertices can be found with reasonable efficiency.

We recognize that there are risks in installing a Si vertex detector along with the strong physics benefits. We feel that an aggressive R\&D program is indicated in this case because the potential physics return is so high. As soon as the above milestones are reached, we shall move forthwith to installation of the Si vertex detector so that physics studies using it can begin during FY88. Te are seeking the endorsement by the PAC of this plan to develop a Si vertex detector.

A summary of the estimated costs for these upgrades is given in Appendix F. An optimum funding profile is also given there. Note that the line for facility improvements has been discussed with Fermilab and the DOE at our last DOE Review. This represents the minimal equipment support needed for routine development and upgrading of CDF.

The first three upgrades discussed above can be viewed as extending the capabilities of CDF within the general scope of the base detector design. For example, the muon supertoroids and TRD fill out the lepton coverage over the full CDF acceptance. On the other hand, the Si VTX detector will add a significant new capability to CDF. 
Another upgrade that has been studied is to increase the amount of absorber and tracking outside the central calorimeters for improving muon identification in the central region of CDF. Such a system appears feasible, but we have decided not to propose this upgrade until after we have actual running experience with CDF.

Fermilab is considering an improvement program to increase significantly the luminosity of the Tevatron Collider. We are now studying the impact of this upgrade on CDF. Some modifications to our electronics and trigger systems will be required and these needs will be presented to the PAC when a firm luminosity upgrade plan is established. 


\section{CDF COLLABORATION}

R. Diebold, W. Li, L. Nodulman, J. Proudfoot, R. Rezmer, P. Schoessow, D. Underwood, R. Wagner, A. Wicklund

Argonne National Laboratory

J. Bensinger, C. Blocker, M. Contreras, L. DeMortier, P. Kesten, L. Kirsch, H. Piekarz, S. Tarem

Brandeis University

D. Amidei, M. Campbell, H. Frisch, C. Grosso-Pilcher, J. Hauser, T. Liss, G. Redlinger, H. Sanders, M. Shochet, R. Snider, J. Ting, Y. Tsay

\section{University of Chicago}

M. Atac, E. Barsotti, P. Berge, M. Binkley, J. Bofill, J.T. Carroll,

J. Cooper, C. Day, F. Dittus, T. Droege, J. Elias, G.W. Foster, J. Freeman, I. Gaines, J. Grimson, J. Huth, H. Jensen, R. Kadel, R. Kephart, A. Mukherjee, C. Nelson, C. Newman-Holmes, J. O'Meara, A. Para, J. Patrick, D. Quarrie, S. Segler, D. Theriot,

A. Tollestrup, ${ }^{*} \mathrm{~K}$. Turner, C. van Ingen, R. Vidal, R. Wagner, Y. Yamanouchi, G.P. Yeh, J. Yoh

Fermi National Accelerator Laboratory

S. Bertolucci, M. Cordelli, M. Curatolo, B. Esposito, P. Giromini, S. Miozzi, S. Miscetti, A. Sansoni

INFN - - Frascati, Italy

G. Brandenburg, D. Brown, R. Carey, M. Eaton, A. Feldman, E. Kearns, J. Oliver, E. Sadowski, R. Schwitters, ${ }^{*}$ M. Shapiro, R. St. Denis

Harvard University

*Spokesmen 
G. Ascoli, S. Bhadra, R. Downing, S. Errede, L. Holloway,

I. Karlinger, H. Keutelian, U. Kruse, R. Sard, V. Simaitis, D. Smith, T. Westhusing

University of Illinois

Y. Arai, Y. Fukui, S. Mikamo, M. Mishina

KEK, Japan

W. Carithers, W. Chinowsky, R. Ely, M. Franklin, C. Haber, R. Harris, B. Hubbard, J. Siegrist

Lawrence Berkeley Laboratory

D. Connor, L. Gladney, S. Hahn, N. Lockyer, M. Miller, T. Rohlay, R. VanBerg, J. Walsh, H. Williams

University of Pennsylvania

G. Apollinari, F. Bedeschi, G. Bellettini, N. Bonavita, L. Bosisio, F. Cervelli, R. Del Fabbro, M. Dell'Orso, E. Focardi P. Giannetti, M. Giorgi, A. Menzione, R. Paoletti, G. Punzi, L. Ristori, A. Scribano, P. Sestini, A. Stefanini G. Tonelli, F. Zetti INFN - University of Pisa, Italy

V. Barnes, A. Byon, K. Chadwick, A. Di Virgilio, A. Garfinkel, S. Kuhlmann, A. Laasanen, M. Schub, J. Simmons

Purdue University

S. Belforte, T. Chapin, G. Chiarelli, N. Giokaris, K. Goulianos, R. Plunkett, S. White

Rockefeller University 
A. Beretvas, T. Devlin, U. Joshi, K. Kazlauskis,

N. Pearson, T. Watts

Rutgers University

J. Buchholz, S. Cihangir, D. DiBitonto, F. Marchetto,

P. McIntyre, T. Meyer, R. Webb

Texas A\&M University

F. Abe, Y. Hayashide, M. Ito, T. Kamon, S. Kanda, Y. Kikuchi, S. Kim,

K. Kondo, M. Masuzawa, T. Mimashi, S. Miyashita, H. Miyata, S. Mori,

Y. Morita, T. Ozaki, M. Sekiguchi, M. Shibata, Y. Takaiwa,

K. Takikawa, A. Yamashita, K. Yasuoka

University of Tsukuba, Japan

J. Bellinger, D. Carlsmith, D. Cline, R. Handler, J. Jaske, G. Ott, L. Pondrom, J. Rhoades, M. Sheaff, J. Skarha, T. Winch

University of Wisconsin 
To: $\quad$ CDR

From: Terry Carroll, Tom Devlin

Subject: Level 3 Status

INTRODUCTION

The Level 3 trigger for CDF is a "processor farm", a system of parallel single board computers, each capable of applying selection criteria to events passed onto it by the hardware triggers at Levels 1 and 2 . Level 3 is essential for CDP operation at luminosities of $10^{30} / \mathrm{cm}^{2} / \mathrm{sec}$. At this luminosity, roughly 50,000 interactions/sec are expected. The Level 1 Trigger uses direct analog outputs from the detector electronics to apply thresholds on such quantities as transverse energy, $B t$ in $3.5 \mu s e c$ between crossings.

Thus, it is essentislly free of deadtime. It will pass $10 \%$ of the events 5,000 erents/sec. Level 2, still working with analog information, applies more sophisticated criteria in event selection, e.g. clustering algorithms in the calorimetry. It is expected to reduce the trigger rate by another factor of fifty to about 100 events/sec. Level 2 requires sereral tens of $\mu s e c$ and introduces a deadtime of 10-20\%. Thresholds at both levels are tunable to allow a reasonable balance of selection criteria and deadtime.

The 100 events/sec which pass Level 2 will be digitized (in about $1 \mathrm{msec}$ ) and sent to Level 3 for application of further trigger requirements in software. It is the goal of the level 3 software to reduce the trigger rate to roughly 1 erent/sec for archiving and 0.1 event/sec for "express line" processing. The hardware must have the input/output bandwidths and the processing speed to handle 100 events/sec in and up to 5 event/sec out.

The system chosen for Level 3 is ACP, the Advanced Computer Program, which was developed at Fernilab for offline data analysis, and is now being developed for commercial production, marketing and support. The initial inplementation of ACP aingle-board computers uses the Yotorola 68020, a 32bit, 16.6-YHs microprocessor ( $\mu$ P) with an associated flosting-point coprocessor. Early benchnarks on this board with the Absoft Fortran-77 Compiler indicate. a performance of about 0.6 Vax-780. Boards using other $\mu P$ chips can added to the system as advances in technology occur.

The immediate goal of the Level 3 project is the develop hardware and software necessary for online operation of a prototype system with 10 Vax equiralents of processing porer for the December, 1986, run. A fullyimplemented system with roughly 50 Vax equivalents of processing power is needed for the run in late 1887 . 


\section{SPECIFICATIONS}

A number of requirements have been placed on the Level 3 hardware and software system. Some are dictated by the data rates expected, and others by our goal of making it easy to transport offline analysis algorithms to the online code in Level 3.

1. Level 3 must be fully integrated into the data acquisition system (DAQ). It must be capable of exchanging messages with the Buffer Yanager, a $\mu \mathrm{Vax}$ which controls the flow of data in the DAq.

2. The system must be capable of processing Level 2 triggers at $100 \mathrm{~Hz}$ and support Level 3 output rates up to 5 Hz. This requires a data transfer rate from Pastbus to the ACP processor memory of about 20 YPytes/sec (100 erents/sec, $100 \mathrm{KBytes/erent,} \mathrm{50 \%} \mathrm{duty} \mathrm{factor).}$

3. Basy transport of trigger algorithms from offline prograns to the online environment is essential. Level 3 will provide online filtering with algorithms written in Portran-77. Various general-purpose software facilities used offline will be available in the online environment. Examples of this are: the YBOS memory management system, histogram entry routines, and parts of CERNLIB.

4. The trigger data base for Level 3 , whereby trigger requirements are specified for a given data run by CDF operations crens, will be compatible with those for Levels 1 and 2 triggers.

\section{HARDTARE STATUS}

A Level 3 test system is currently installed on the 9th floor of Wilson Hall immediately adjacent to the ACP development area. The host computer for the system is a Vax-780 (BSNDBG) equipped with a UPI interface to Pastbus. Figure 1 shows the hardware components currently installed and in use for hardware and software development.

The ACP hardware resides in two VR crates (standard, commerciallyavailable crates with high-bandwidth backplanes). High-speed data transfer between the ACP system and other devices occurs over the Branch Bus (BB), a 32-bit bus with appropriate control lines. The nodules, on loan from the ACP group, are:

-- two CPU's, 68020 single-board processors with 2 UBytes memory,

-- a VRY (VE resource module) crate controller,

-- a BVI, Branch Bus to VIE interface,

-- aBC, DEC Q-bus to Branch Bus interface,

-- a QVI, Q-bus to VRe interface, 
-- a hE memory board, MEM, and

-- two Branch Bus terminators, BBT's.

The development of software for the nodes is concentrated in the lower right-hand area of Fig. 1 . It consists of a $\mu \mathrm{Vax}$ which can communicate to the Branch Bus through a Q-bus interface (DRV11-WA). The full package of routines used by the ACP group for creating 68020 programs exists in the $\mu$ Vax (and other computers DECnetted to it). These include the Absoft Fortran-77 compiler, linkers and debuggers.

The CDF hardware development (upper left-hand area of Fig. 1) is focused on the FBBC, an interface between Fastbus and the Branch Bus. This is the channel for passing events from CDF to the ACP system, and is a critical-path item for the December, 1986, run.

Tests of the FBBC are controlled from the Vax-780 by an interactive program which allows the user to select any of a number of operating modes. Single-word and block transfers can be executed from the Vax memory through a data path which includes the UPI interface to Fastbus, the FBBC to Branch Bus, BVI to the VRE backplane and into the CPU memory.

Transfer through the UPI is rather slow, and cannot test the full bandwidth of the FBBC-BVI combination. In order to test the system at high rates, the same interactive test routine includes the option of setting in motion an identical set of data transfer tests driven by a Fastbus-resident processor, the SSP. Test summaries and error logs are reported back to the Vax in the same form as its own tests. In addition to its advantage of higher speed, the SSP can run for extended periods without degrading the performance of the host Vax.

The SSP can currently block transfer to memory in 68020 nodes at a rate of $13 \mathrm{KBytes} / \mathrm{sec}$ with error rates of about 1 per million 32-bit words on average. Power, cabling and grounding are being cleaned up as a first step in eliminating the 1ow-level error rates. The SSP has also executed pipelined writes to node memory at a rate of $18 \mathrm{MBytes} / \mathrm{sec}$, - close to the design goal of $20 \mathrm{MBytes} / \mathrm{sec}$.

CDF currently has two wirewrap FBBC modules. The design is quite stable, and during recent weeks, the BVI has been the subject of most hardware modifications to support high data transfer rates. This the first opportunity for the ACP development group to test the BVI at these rates, and they will produce an upgraded version of the BVI when these tests are complete. $\Lambda C P$ is also interested in producing a printed-circuit or multi-wire version of the FBBC.

SOFTTARE DEVELOPMENT: CONTROL OF FASTBUS

For the current tests, subroutines have been written in Fortran to control various control and data transfer operations involving the FBBC. They have been coded in the standard CDF structure for compound Fastbus operations. 
They generate commands for the Fastbus interface (UPI) which can be executed immediately, or incorporated into longer lists of UPI operations for more efficient execution. These should be useful without change in the main CDF online programs.

The interactive test program for the FBBC described in the previous section should continue to be useful for in situ tests of the FBBC and processor farm after it is moved to $B O$. Standard CDF software packages were used for user menus and memory management. The database defining the farm and the status and addresses of each node in the farm is the same as that which will be used by the online programs. The test program contains facilities for adding and deleting processors from the farm inventory, and testing the full system or any part of it.

\section{SOFTWARE DEVELOPIENT: GENERAL FACILITIES}

A number of software packages have been transferred directly or rewritten to run in the ACP node environment. The library of routines for the YBOS memory management systems in now operating in ACP. $A$ number of routines from CERNLIB and UTIIIB have been transferred to ACP. Some were originally in VaxYacro, and had to be re-written in Fortran or in 68020 assembly language.

SOFTWARE DEVELOPMENT: TRIGGER ALGORITHMS

A prototype jet algorithm has been written for the nodes' and tested with data generated by a Yonte Carlo simulation of CDF calorimeter data in standard YBOS format. The algorithm decodes the raw data banks, searches for transverse energy clusters in $y-\phi$ space (rapidity and azimuthal angle) and calculates jet parameters.

The algorithm has been run in both the real 1 CP nodes and in a node simulator which actually executes on a Vax. The latter was useful for implementing the Host/Node structure, but was immune to certain Vax/68020 differences.

Although errors in the Absoft Fortran-77 compiler have required some kluges, the trigger algorithm is fully operational in the nodes. A comparison with the Vax simulation shows identical results for the central calorimetry data. Benchmark comparisons between 68020 and Vax operation are being performed at this writing.

A new release of the Absoft compiler is now available. The trigger algorithm and associated libraries will be recompiled to eliminate the known bugs and kluges.

FUTURE HARDWARE DEVELOPLENT REQUTRED

In parallel with Level 3 another group is developing a Fastbus device call the Event Builder to assemble in one place the data from the many devices 
which collect information from the various CDF detector subsystems. It is essential to high-speed data collection and transfer to the Level 3 processor farm.

The hardware Event Builder is unlikely to be ready before early 1887 . Therefore, until it is ready, its function in the data acquisition system will be performed in the host Vax by software. Under these conditions, Level 3 will be served by a "consumer process" in the Vax which copies events from the data stream and transfers them back through Fastbus to the FBBC and the processor farm. This mode restricts Level 3 to a role as a monitor of accepted events, rather than an active data filter. It does constitute an extremely valuable opportunity to integrate Level 3 into the data acquisition network, to simulate the Level 1/hevel 2 operation and check consistency between the trigger hardware and corresponding software model.

Level 3 should be prepared for the possibility that the Event Builder becomes operational early in 1887, prior to completion of that run.

A fully-integrated hardware Event Builder and Level 3 system is scheduled for the run later in 1987, and should be prepared for full luminosity. At this stage, two complete Event Builders are required, each served by a separate FBBC interface to the ACP processor farm.

Another important piece of hardware for high-speed operation is the Bus Switch to be built by the ACP group. This device will permit the Level 3 processor farm to be served by several parallel Branch Buses in order to increase bandwidth. It will allow simultaneous data transfer from each FBBC to the farm, and output of data from the farm to the data-logging system.

PERSONNEL

The following personnel are now working on the Level 3 project:

$\begin{array}{lll}\text { Andy Beretvas } & \text { Rutges } & 30 \% \\ \text { Terry Carroll } & \text { Fermilab } & \\ \text { Tom Devlin } & \text { Rutgers } & 50 \% \\ \text { Brenna Flaugher } & \text { Rutgers } & \\ \text { Ping Hu } & \text { Rutgers } & \text { (Summer) } \\ \text { Umesh Joshi } & \text { Rutgers } & \\ \text { Yark Larwill } & \text { Fermilab } & \\ \text { Youhei Morits } & \text { Tsukuba } & 50 \% \\ \text { Zhizhong Zhang } & \text { Rutgers } & \text { (Summer) }\end{array}$

Terry Watts is beginning a sabbatical leave from Rutgers and will spend about $50 \%$ of his time working on Level 3 compatibility with Level 1 and Level 2 triggers.

Irwan Sidharta, a-Rutgers E.E. graduate, will spend, the summer at Fermilab working with the group developing the Event Builder.

Ping Hu will complete his course work at Rutgers during the Fall semester and rejoin this project full time in December, 1986. 
Bob Hollebeek from Penn has expressed interest in working on Level 3 . Mark Larwill is supported in the development of the FBBC by other members of the technical staff of the data systens group. We have received a great deal of help from many members of the ACP group: Glenn Case, Rick Hance, Hari Areti and Art Cook in hardware development; Irwin Gaines, Joe Beal, Mark Fischler in software development. The overall interest, support and encouragement provided by Tom Nash has been very important.

\section{SCHEDULE AND MILESTONES}

1-Jun-86 Prototype trigger algorithm studies complete

20-Jun-86 FBBC development complete

1-Jul-86 Start installation of 2-crate processor farm with FBBC at CDF

31-Jul-86 Conversion of $\mu \mathrm{Vax}$ management of processor farm to online environment, interface to Fastbus and exchange of messages with data acquisition controller

11-Aug-86 Vove $\mu$ Vax to CDF

15-Sep-86 Complete development of "consumer process" to copy events from data stream to Level 3 processor farm

Dec-86 Beam. First operation of Level 3 with real events.

Early 87 Hardware Event Builder operational. Possible tests of Level 3 at high speed with real events before end of current run, processing power $\sim 10$ Vax-780 equivalents.

Bus Switch complete

Fall 87 Full implementation of Level 3,>50 Vax-780 equivalents. 


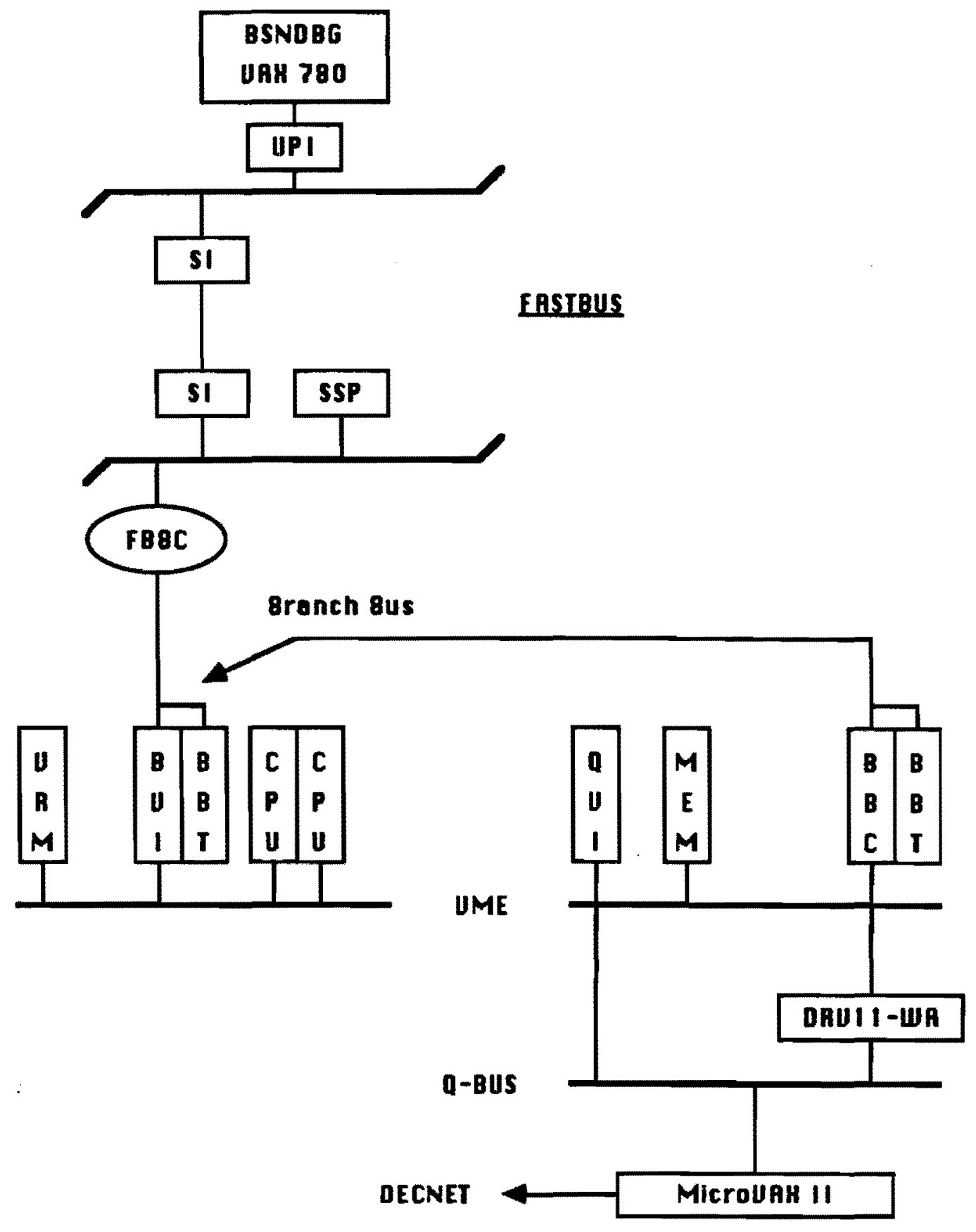

Fig. 1: LEVEL 3 TEST SYSTEM 
TO: Dennis Theriot

May 22, 1986

From: Terry Carroll

Subject: Level 3 CostEstimate

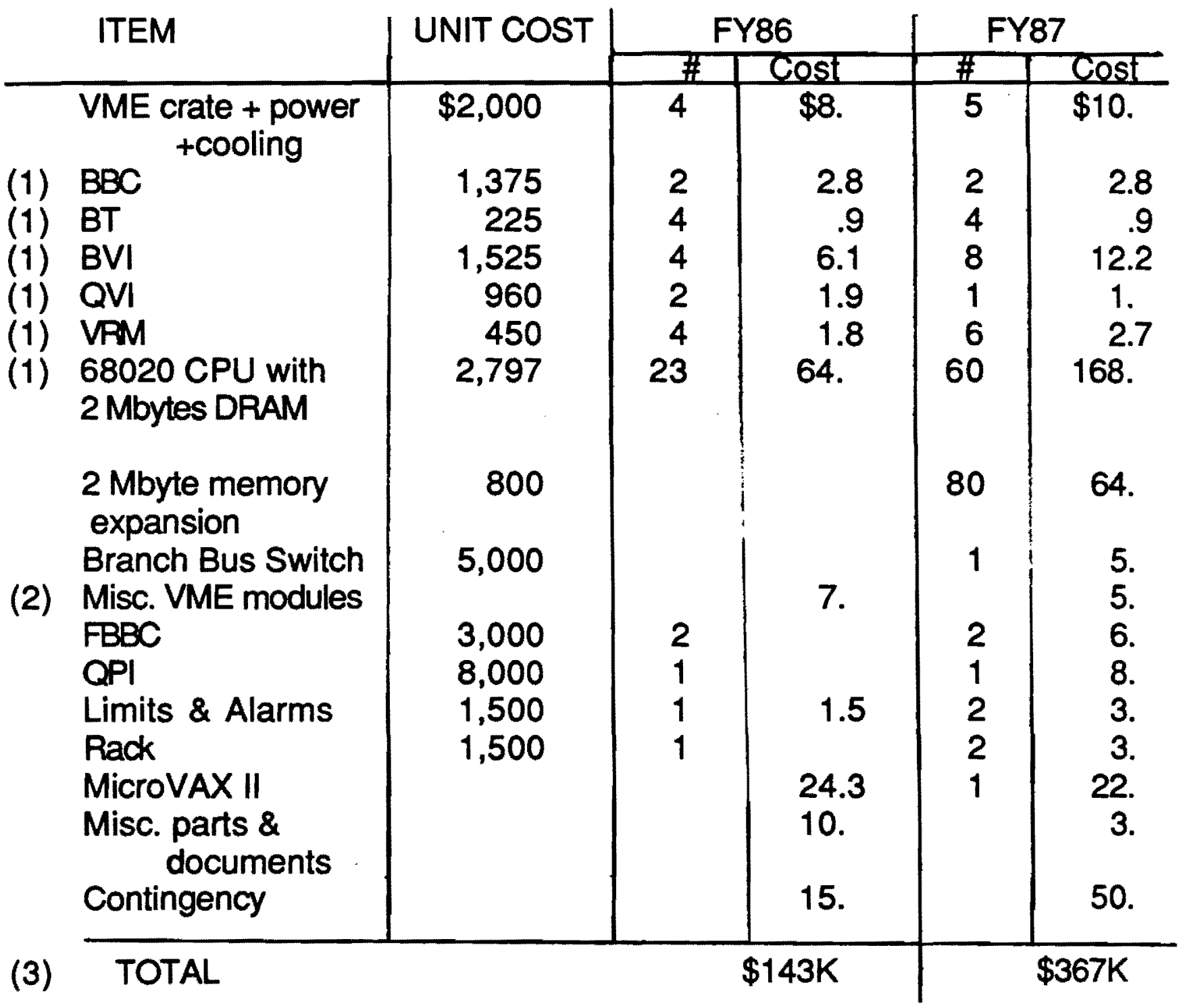

(1) Prices are quotes from OMNIBYTE in West Chicago for operational modules with a 2 year warranty.

(2) VME display modules, extender cards, memory modules, etc.

(3) Estimate for FY86 includes funds from Tsukuba University and $\$ 36 \mathrm{~K}$ from Rutgers. 
Appendix C

20 May 1985

SUPERTOROIDS FOR CDF:COMPLETING THE MUON COVERAGE

\author{
D. Carlsmith, D. Cline, R.Handler, A. Jaske, G. Ott, \\ L. Pondrom, J. Rhoades, M. Sheaff, J. Skarha, T. Winch \\ University of Wisconsin, Madison, WI
}

D. Theriot

Fermilab, Batavia, $I L$

\begin{abstract}
It is proposed to complete the CDF muon coverage with a single toroidally magnetized spectrometer in the shape of a sunflower at each end of the central detector. The system would provide a high $\mathrm{Pt}$ muon trigger and momentum analysis over the range 16 to 55 degrees in polar angle.

\section{INTRODUCTION}

The proposed supertoroid system would complete the muon detection capability of the CDF detector from a polar angle of 3 degrees to 177 degrees relative to the circulating protons, corresponding to $+/-3.6$ units of pseudo-rapidity. In its present form the CDF detector has a gap in muon coverage between 17 and 55 degrees, or 1.25 units of pseudorapidity on each side of the collision point. This gap represents $35 \%$ of the total 7.2 units of rapidity in the central region of phase space accessible to CDF.

The physics capability of the CDF detector is substantially increased by this improvement in muon coverage. The identification and momentum measurement of single muons are important for missing ET measurements, for the study of $W$ decays, and for tagging heavy flavors. Single muon detection increases linearly with phase space coverage. The efficiency of the detector for multi-lepton events increases faster than linearly because of the correlations among the sources of several prompt muons. Hence the completed muon coverage between 3 and 177 degrees has a very high priority as a detector upgrade.

The scope of this proposal is similar to previous proposals to provide 1) a toroidal spectrometer for the range 16-32 degrees and 2) additional unmagnetized iron and drift chambers patched on the central detector for the range 32-55 degrees, but is an integrated system with momentum analysis for the entire angular region 16 to 55 degrees. The system provides additional absorber for muon identification and an independant momentum measurement which may be compared with the measurement in the central tracking chamber for purposes of background rejection.
\end{abstract}




\section{MAGNET DESIGN}

Figure 1 shows a preliminary design of the magnet. At intermediate angles, the sunfower shape is natural: particle trajectories are normal to the bend direction and to the drift chambers, and detector area is minimized. Below 30 degrees, the magnet is $\mathbf{4 0}$ inches thick and has the shape of a standard toroid with a projective inner surface. The central 16 degree hole for the forward calorimeter could accomodate a previously proposed transition radiation detector. A conical shape for polar angle greater than $\mathbf{3 0}$ degrees is approximated by flat 'petals', 32 inches thick, with machined mating surfaces. Each petal covers 30 degrees in azimuth and weighs about 48 tons.

The geometry of the CDF detector and collision hall and the need to extract the endplug imply that any system in this angular range must be carefully sculpted. $A$ number of interferences must be specially dealt with :

1) The largest interference is with the floor. We propose that steel be embedded in the floor to provide a smooth path for the magnetic flux in the petals of the sunflower. Whether this region (approximately $20 \%$ of the solid angle of the supertoroids) would be instrumented is under study.

2) The top 20 ton of the magnet does not fit in the door. We propose to assemble as much as fits in the assembly area and piece on the top in the collision hall under the assumption that the sunflower would not often be removed to the assembly area.

3) The I-beam supports for the beam-beam counters would be cut off and the counters supported from the sunflower inner surface.

4) The arms which support the plug would be cut off and an independant support system ('ATLAS') would be brought in for plug extraction. A reduced thickness of steel in the petals is nevertheless required to clear the plug supports at 0 degrees in azimuth.

5) The magnet would split in the center and each half would be pulled out and around the central detector for plug extraction.

6) The dewar would be moved.

The magnet would be saturated by four water-cooled coils with $40 \mathrm{Kamp-turns} \mathrm{per}$ coil of design similar to those used for the forward toroids. The design field varies from $16.5 \mathrm{Kg}$ at the inner radius to $19.5 \mathrm{Kg}$ at the outer radius.

\section{CHAMBERS}

We propose a double plane of electrodeless chambers (Figure 2), similar to the forward muon chambers, on the front and rear surfaces of the sunflower magnet. Chambers are arranged in the form of a pinwheel with each sense wire tracing out a 30 degree chord of a circle. The track position in azimuth is measured by 5 degree pads. As in the forward system, the cells are graded (proportional to polar angle), and project to the nominal interaction point, to provide for a Level $1 \mathrm{Pt}$ threshold on the basis of a coincidence of hits in the front and rear chambers. The rear plane of chambers is set 10 inches off the magnet face to maximize analyzing power.

Given an accurately known vertex position, the momentum of the muon is determinable from the entrance and exit points with a multiple Coulomb scattering limited resolution of $\approx 20 \%$. The central tracking chamber should measure the momentum with better resolution over most of the solid angle. Two planes are sufficient to provide a Level 1 muon trigger and a momentum measurement which may be compared with the central 
tracking measurement.

\section{TRIGGER}

A trigger similar to the $300 \%$ trigger of the forward system would consist of the OR of the set of coincidences of cell $I$ in the front chamber with cells $I-1, I$, and $I+1$ in the rear chamber. To provide a $\mathrm{Pt}$ threshold at Level 1 similar to the forward muon system requires 118 trigger cells at about .011 units of pseudo-rapidity per cell, roughly twice the number of trigger cells in the forward system. The minimum and maximum drift times are $330 \mathrm{~ns}$ and $1116 \mathrm{~ns}$ respectively. In so far as the field of the solenoid is cylindrically symmetric, angular momentum conservation requires that a particle exit the flux return path pointing at the origin, rotated somewhat in azimuth, so unless central tracking information is used in the trigger, the solenoid field has no visible effect.

This trigger scheme is potentially compromised by the expected vertex distribution: the projective cells can not simultaneously image a distributed source efficiently and provide a high Pt threshold. Consider an infinite momentum track which passes through a fixed cell in the front plane of chambers. As the production point shifts away from $z=0$, the projection through the front chamber to the rear chamber shifts out of the projective cell. The displacement in units of cells at the rear chamber is approximately 1.4 cells per foot of displacement in z. To good approximation, the displacement in units of cells for graded cells is independant of polar angle.

This suggests a modified trigger which incorporates vertex information from the beambeam counters. The rear chamber trigger bits are latched in a long shift register and shifted by an amount depending on the vertex position prior to forming the $1^{*} 3$ coincidences. The vertex position is provided at Level 1 by a simple meantimer circuit.

An idealized Monte Carlo simulation was made to study this trigger scheme. The sunflower and calorimeters were approximated as pieces of spherical shells with an average of 70 radiation lengths assumed for the calorimeters. The vertex resolution of the BBC meantimer was assumed to be 6 inches and was digitized in bins of 6 inches. In Figure 3, we show the trigger efficiency (including edge losses) as a function of $\mathrm{Pt}$ for a gaussian vertex distribution with the nominal r.m.s. of 1 foot, with and without the vertex correction. The improvement in the threshold function is limited by the crude resolution assumed for the Level 1 beam-beam counter information. The relatively high asymptotic efficiency after the vertex correction was found to be maintained for a vertex distribution with a sigma of 1 meter which implies that the trigger would be sensitive to interactions virtually anywhere on the axis of the central detector.

\section{BACK GROUNDS}

Three types of backgrounds may be anticipated: cosmic rays, pion and kaon decay in flight in the central tracking volume, and leakage from showers in the calorimeters (including decays of particles generated in the hadronic cascade). We present below rate estimates for each of these backgrounds.

An estimate of the cosmic ray background is found using the flux

$$
\frac{d N}{d \Omega}=I_{0} \sin ^{2}(\theta) \sin ^{2}(\phi)
$$

and the trigger solid angle

$$
\Delta \Omega \simeq 3 \times \text { cell area } / t^{2}
$$


where $I_{0}=5 \times 10^{-3}\left(\mathrm{~cm}^{2}-s t r-s\right)^{-1}(p>2 \mathrm{GeV} / \mathrm{c}), t$ is the chamber separation and the cell area depends on polar angle. Integrating the flux times the solid angle over the top half of a sunflower and assuming that a trigger in the bottom half would have triggered the top half of the other sunflower, we find for 3 bunch operation at a luminosity of $10^{30}\left(\mathrm{~cm}^{-2}-\mathrm{s}^{-1}\right)$ :

Rate $=558 \mathrm{~Hz} * 2$ ends * $(.171$ live time $) *(.5$ probability of a BBC hit per Xing $)=$ $95 \mathrm{~Hz}$.

This rate could be reduced by a factor 6 if the azimuthal bite of a trigger cell were restricted to 5 degrees by incorporating the pad information into the trigger. Most of the cosmic rays which satisfy the trigger are at large polar angle where some rejection is expected from the requirement that a matching track be found by the central track processor.

We estimate the punchthrough and decay in flight using the single charged hadron inclusive production cross section from UAl:

$$
\frac{d \sigma}{d P_{t} d Y}=\frac{2 \pi A P_{t}}{\left(1+\frac{P_{t}}{1.3 G e V / c}\right)^{n}}
$$

where $\left.A=0.47 \mathrm{barn} /(\mathrm{GeV} / \mathrm{c})^{2}\right)$ and $n=8.07$ is the power extrapolated to $2 \mathrm{TeV}$ using both ISR and collider data. (see PL 118B,169 (1982).)

To estimate the trigger rate from decay in flight, we numerically integrate the production cross section times a luminosity of $10^{30}\left(\mathrm{~cm}^{-2}-\mathrm{s}^{-1}\right)$ times the trigger efficiency as a function of $P_{t}$ described above, times the decay probability assuming $20 \%$ kaon content with .63 branching fraction to muons and a conservative angle independant decay length of 2 meters. The resulting rate estimate is $4.4 \mathrm{~Hz}$ per sunflower. This rate should be regarded as an order of magnitude estimate as it is extremely sensitive to the trigger rejection at low $\mathrm{Pt}$ and to the shape of the production distribution.

The momentum measurement by the sunflower should aid in the rejection of the problematic 'seagull' decays of low energy pions and kaons which fake high $\mathrm{Pt}$ tracks in the central tracking chamber.

For under 3 meters of iron equivalent, shower leakage is dominated by hadronic punchthrough rather than by real muons from decays of shower particles (see F.S. Merrit et al., NIM A245,27 (1986)). To estimate the trigger rate from shower leakage, we use the Snowmass ' 84 parameterization of the probability of greater than zero particles exiting an absorber length $\mathrm{x}$ of $\mathrm{Fe}$ equivalent:

$$
P(x, p)=0.13 p^{1.62} e^{-x / 23 c m}
$$

Integrating the single charged hadron production rate times the leakage probabilty for a fixed absorbtion length of 2 meters Fe equivalent over the angular range of the sunflower, we obtain a rate of $5.5 \mathrm{~Hz}$ of leakage events at the rear of the each sunflower. The leakage rate into the front chambers is a factor of roughly 75 higher. All leakage events may not satisfy the trigger. However, the presence of a leakage particle in a rear chamber is likely to be associated with hits in more than one cell in the front chamber so the effect of the $\mathrm{Pt}$ threshold trigger is difficult to estimate without a detailed simulation of hadronic cascades. 
A multiplicity cut in the front chamber could be used to eliminate such events. For the remaining events, $2 \mathrm{GeV} / \mathrm{c} \mathrm{Pt}$ threshold reduces the trigger rate due to leakage by a factor of 10 .

costs

An estimate of the cost is obtained by scaling the costs of the present toroids. The surface area of a sunflower is about $2300 \mathrm{ft}^{2}$, approximately 1.9 times the chamber area of three forward muon chamber planes. Each magnet is 812 tons, approximately equal to the volume of a toroid magnet pair. We require 8 coils with about 1600 feet of conductor per coil. For 118 trigger cells in polar angle, assuming half as many cells to resolve the ambiguity, with each cell covering 30 degrees in azimuth, each sunflower requires 4248 TDC channels. For 5 degree azimuth pads, dividing the polar angle into 10 regions, there are 1440 pads per sunflower. We then find the following estimates (both ends together): Cost estimates(kilodollars)

\begin{tabular}{|c|c|c|}
\hline Magnet Design & & $\$ 100 \mathrm{k}$ \\
\hline Magnet & & 1590 \\
\hline Coils & & 300 \\
\hline Base & & 200 \\
\hline Power Supply & & 100 \\
\hline Chambers & & 558 \\
\hline ADC's & $2880 \mathrm{ch} * \$ 100 / \mathrm{ch}$ & 288 \\
\hline TDC's & $8496 \mathrm{ch} * \$ 100 / \mathrm{ch}$ & 850 \\
\hline Total & & $83986 k$ \\
\hline
\end{tabular}

The total does not include the floor modification or the endplug support ATLAS. 


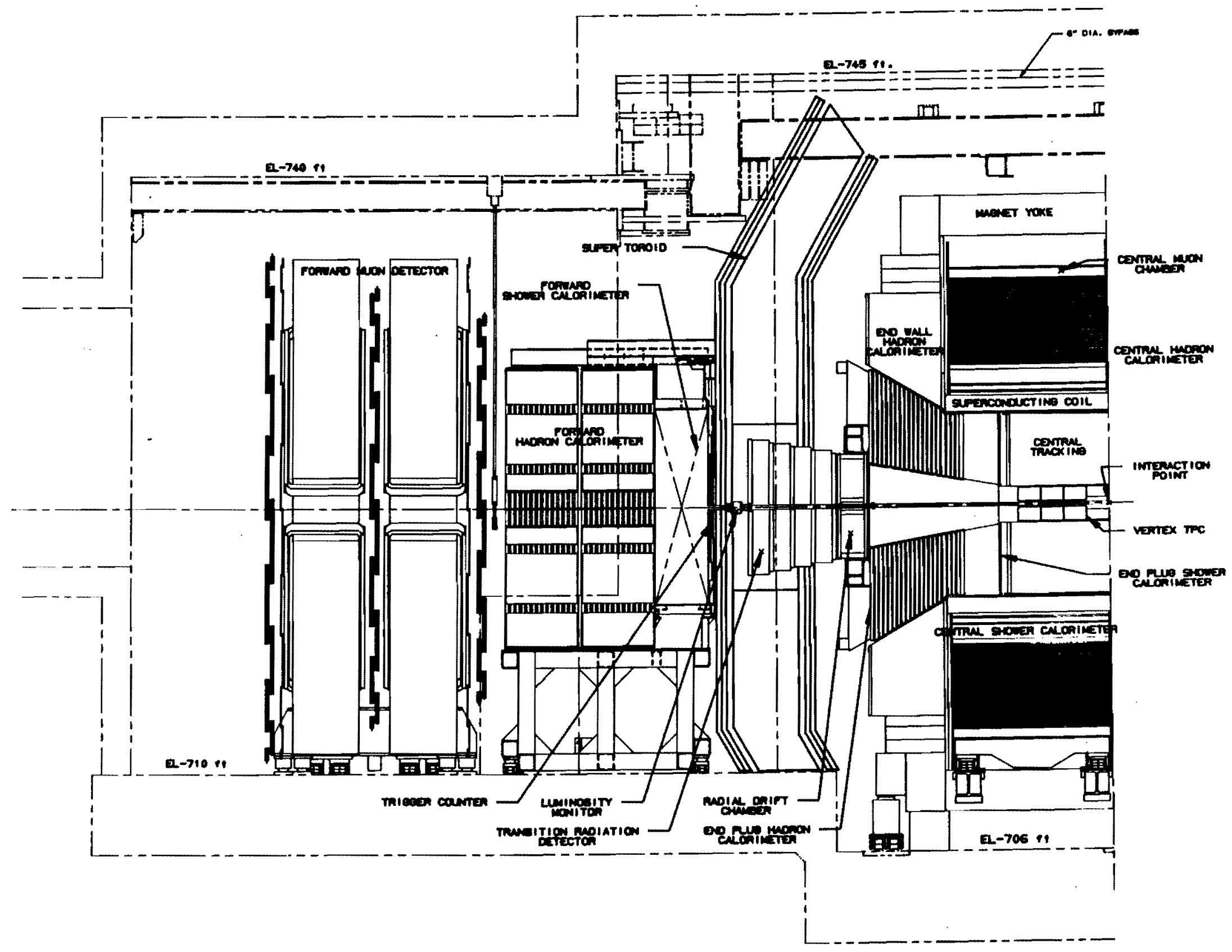


Frouke 2

Supertoroid Drist Chambers (sront Sace)

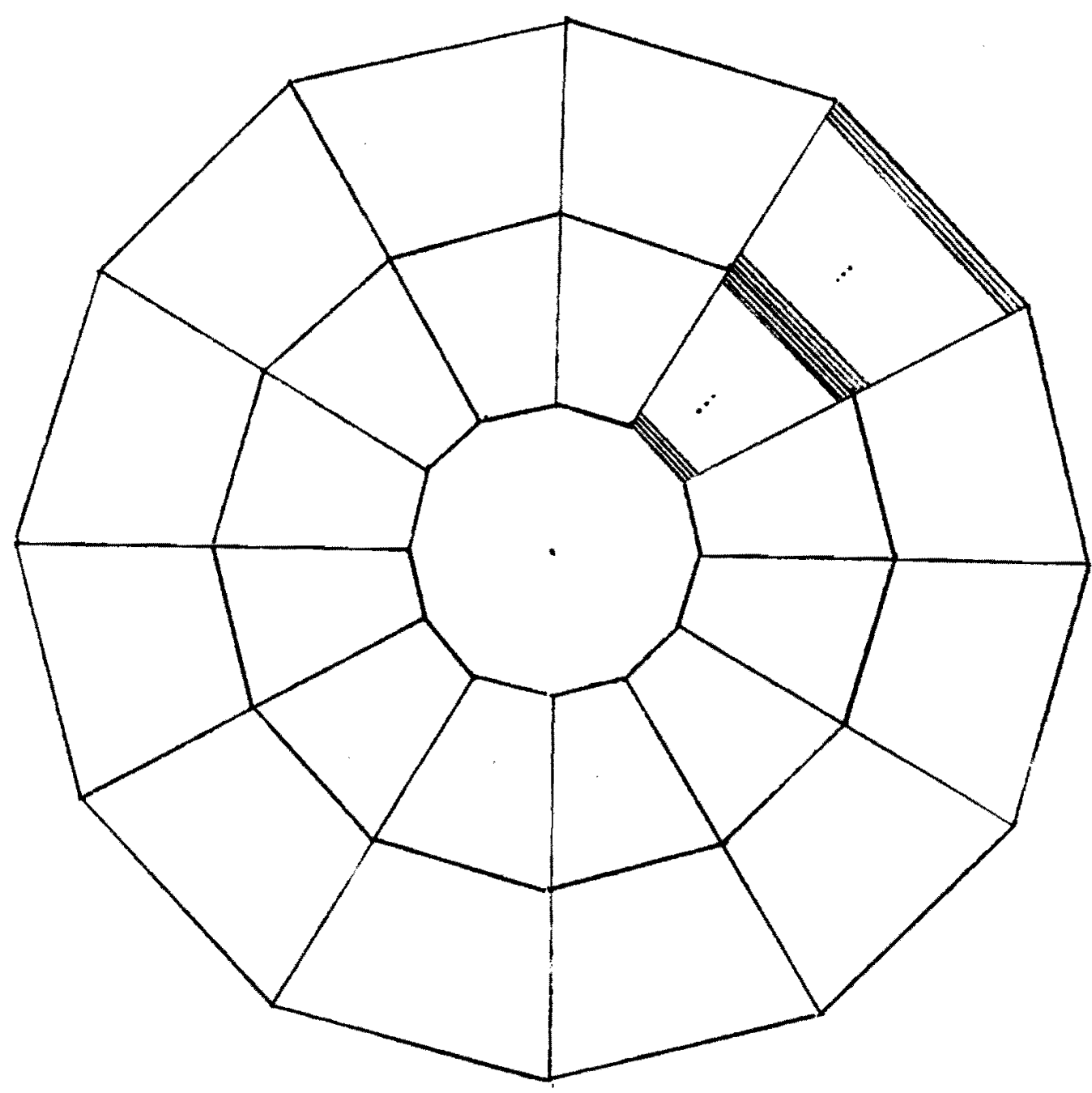

Figure 2 
$7+4 x-100$ co: cos

The 1

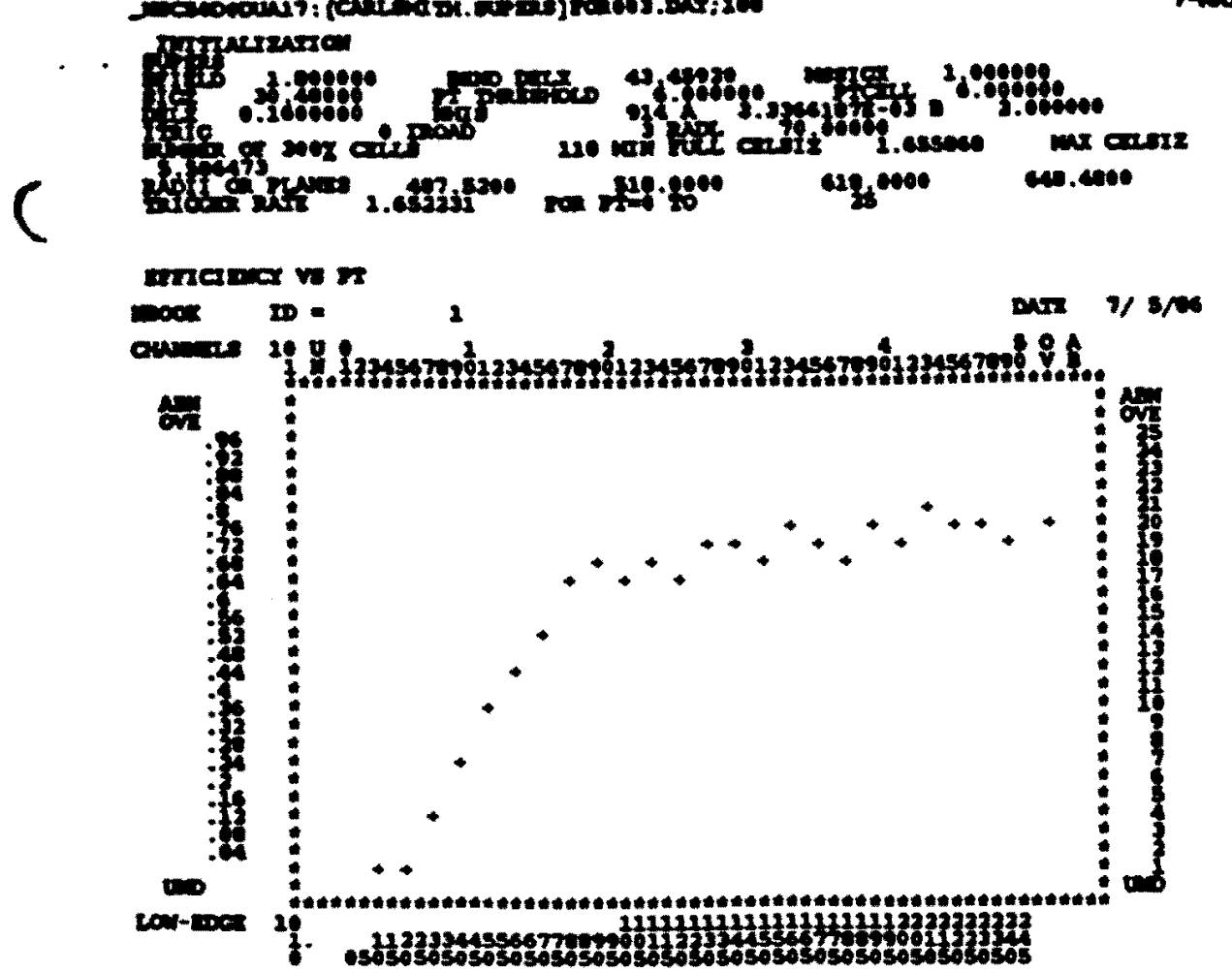

$300 \% \quad 118$ cells no sec conation

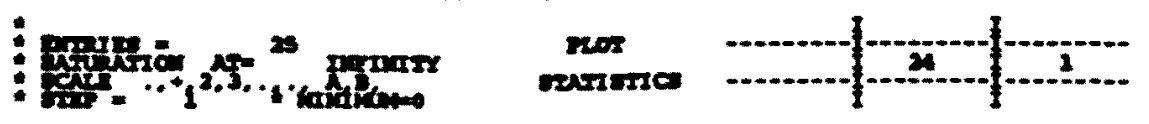

maxpertar

C 
Appendix D

\title{
A Proposal to Bulid a Transition Radiation Detector System for the Forward Angle Region of CDF
}

\author{
G. Brandenburg, M. Atac, J. Bensinger, E. Sadowski
}

\section{Introduction}

We propose to add a Transition Radiation Detection system in the forward/ backward angle region of the Collider Detector at Fermilab (CDF) to improve electron identification. In this region such devices are ideally suited to the physics needs of CDF and to the geometrical space available. This region has no magnetic field to aid in particle identification, yet there is sufficient space along the beam line for a low mass TRD system. Such a system would not intefere with any existing CDF components.

The current version of the CDF detector has tracking chambers, calorimetry, and muon detectors covering most of the available solid angle. At $2 \mathrm{TeV}$, the 176 degrees of polar angle which are covered are totally within the "central region". Specifically, the total rapidity range of $2 \mathrm{TeV}$ interactions is approximately \pm 7.5 units, while the CDF detector covers \pm 4 units. Thus the forward/backward region $\left(2^{\circ}-10^{\circ}\right.$ at either end), which covers the pseudorapidity range of 2.4-4.0, is an integral part of the the central region. The forward/backward region already has good muon detection efficiency; $a$ TRD system in this region would provide electron identification with similar precision.

Good lepton identification is essential for much of the interesting physics available to CDF. In particular the production of heavy quark flavors, of W's and Z's, and "new physics" such as Higgs bosons will all have distinctive lepton signatures. The importance of good coverage of the small angle region is seen in Fig. 1, which shows the rapidity distribution of the decay leptons from W's produced at $2 \mathrm{TeV}$. It is clear that the identification and measurement of both electrons and muons is required to the smallest lab angles possible. In a more general sense the decay products of any object with $\sqrt{s}=80 \mathrm{GeV}$ will extend to rapidities of \pm 4 . It is also possible that the producton of heavy quark pairs may be enhanced by a diffraction-like mechanism at small angles and that their decay products would populate the forward region.

For the first CDF run the various detectors will be deployed as shown in Fig. 2. The smallest angles are covered by the forward tracking chambers (FTC), the Forward Electromagnetic and Hadron Calorimeters (FEMC and FHC), and the Muon Toroids. The existing calorimetry in the forward (and backward) region has a granularity of $\Delta \eta=0.1$ and $\Delta \theta=5^{\circ}$. The tower structure of these calorimeters is diagrammed in Fig. 3. The combination of FEMC, FHC, and FTC is able to adequately measure the position and energy of isolated electrons, provided they can be identified. However, because there is no appreciable magnetic field in this region, this identification must rely on the relative amounts of energy deposited in the three depth segments of the calorimetry, correlation of tracking information and shower centroids, and the width of the showers. 
The lack of effective momentum measurement in the forward direction prevents using a comparison of the measured track momentum to calorimeter energy for electron identification. This comparison when used in the rest of the detector is an effective way of identifying the low pt pion plus photon background. We estimated that a TRD will give us an improvement of a factor of 10 to 50 over what we can do with the CDF fringe field alone. An improvement of this order is essential for such things as identifying semileptonic decays of heavy quarks.

A pion can masquerade as an electron in one of several ways: (a) a low momentum charged pion overlaps with with a $\pi^{*}$, (b) a $\pi^{*}$ Dalitz pair is not properly correlated, (c) a charged pion produces an early electromagnetic shower in the calorimetry. Eliminating (a) and (b) currently depends on the the ability to match tracking information and shower positions. The problem of charged pion shower development , (c), is addressed in Figure 4, which shows the total energy deposited by $60 \mathrm{GeV} \pi$ 's and e's in the Forward Electromagnetic Calorimeter. Figure 5 shows a correlation plot of the energy deposited in the two depth segments of the EM calorimeter for the same particles. Cuts were made on the ratio of EM energy to total energy, the ratio of the front half of the EM calorimeter to the total EM energy, and the shower width. For the test beam case shown here there was no hadron calorimeter data, so the measured beam momentum was used for the total energy. The table below shows that after all three cuts the $\pi$ survival probability is less than $1 \%$ for reasonable e efficiencies. In a colliding beam environment, the total $\pi$ survival probability will be about $1 \%$. This is clearly not good enough if we need to uniquely separate electrons from the much more prolific pion background. A transition radiation detection system would improve the electron identification capabilities for both cases (a) and (c).

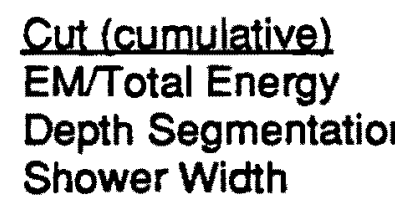

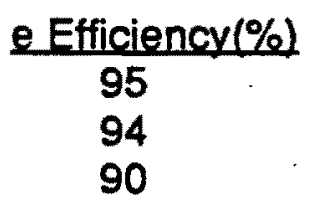

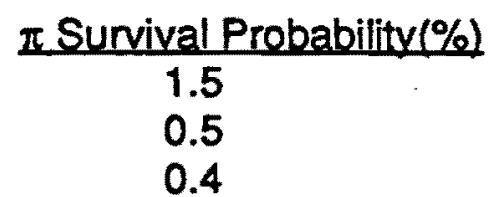

The Forward Tracking Chambers are dritt chambers with radial wires. This design gives an excellent measurement of the azimuthal coordinate, but a poor measurement of the polar angle. This will be mitigated somewhat by implementing current division on the wires. It has been proposed to build a second set of tracking chambers with azimuthal wires ("theta chambers") to provide an accurate orthogonal coordinate. Measuring both coordinates well will be important in cases (a) and (b) above for matching trajectories with showers in the FEM. We propose to build the TRD system with chambers that are similar in design to the theta chambers, and which will serve the dual function of measuring particle trajectories and detecting transition radiation.

The proposed TRD system is located approximately $5 \mathrm{~m}$. from the interaction point. At this distance particles are separated sufficiently to be separately identified. In particlular the typical separation of particles inside a jet will be $-4 \mathrm{~cm}$. at the inside edge of the detector. 


\section{Transition Radiation Detectors}

The radiation of low energy photons as extremely relativistic particles pass through the successive layers with different indices of diffraction is strongly dependent on the Lorentz factor $\gamma=1 / N\left(1-B^{2}\right)$. It saturates at $g \approx 10^{4}$. It is possible to enhance this effect by building a radiator which consists of many successive thin sheets of very lightweight material. Figure 6 shows the average number of TR photons produced when electrons and pions pass through a block consisting of $=200$ sheets of $17 \mu \mathrm{m}$. thick polypropalene. For electrons with energies above $5 \mathrm{GeV}$ the number of expected photons is constant at approximately two. The number of TR photons for pions in the same energy range is seen to be much less than one.

A transition radiation detector system which consists of several modules of radiator and photon counting chambers will maximize the number of observed TR photons by decreasing the amount of material that a photon must traverse before detection. A Russian group has built a detector which consists of twelve such modules, each consisting of a 210 sheet radiator block and a photon-counting proportional chamber. Figure 7 shows the number of TR photons detected for $\pi$ 's and $e$ 's in the $10-80 \mathrm{GeV}$ region. On the basis of this distribution they obtain a $\pi$ rejection factor of 1500 with an electron inefficiency of $0.5 \%$.

In CDF we have a space in front of the forward calorimeters which is approximately $1.5 \mathrm{~m}$. in length. This is sufficient room for a four module TRD system. Based on the TR photon output for a single module shown in Fig. 6, we can estimate the pion survival rate as a function of electron innefficiency for such a four module system. This is shown in Fig. 8 . It is predicted that less than $2 \%$ of pions will be misidentified as electrons with an electron inefficiency of less than $2 \%$. This factor of 50 is independent of the factor of 100 obtained from the calorimeter depth segmentation, resulting in an overall $\pi$ rejection factor of $=5000$.

\section{Construction Details}

The transition radiation detection system we are proposing consists of four radiator blocks and five drift chambers as illustrated in Fig. 9. The system will immediately follow the FTC package. The proposed Muon Supertoroids are omitted in the drawing: if they are present the system will fit inside their aperture and no additional support strut will be necessary. The TRD system will be moved to the side together with the beam pipe if the end plug is to be removed. 
The radiator blocks have an octagonal cross section to the beam and extend from the beam pipe to just beyond the ten degree cone. They consist of 250 sheets of $13 \mu \mathrm{m}$. mylar separated by $1 \mathrm{~mm}$. and stretched between $\mathrm{G} 10$ frames. The sheets are randomly dimpled so that they maintain their separation from each other. This makes it possible to eliminate all framework from the "active area" of $2^{\circ}-10^{\circ}$. A method of dimpling the sheets with a warm rounded iron has been successfully tested. It is preferable to build each radiator block as a single unit not only to eliminate framework, but also because it is much easier to stretch the mylar sheets from a symmetrical frame. The mylar sheets are glued to the the frame with contact cement making it possible to add several sheets to the structure each day. It is also necessary to glue two strips of mylar together to form a wide sheet: commercially-available rolls will not cover the entire frame.

The drift chambers are proposed to have wires strung in the "theta chamber" geometry - see Fig. 10 . There will be approximately 30 sense wires extending from $2^{\circ}$ to $10^{\circ}$. These wires will be read out independently for each octant resulting in 240 signals per chamber. The frames of the chambers will be buit from lightweight materials such as hexcell wherever possible. It will be necessary, however, to have an outer aluminum support structure. The chambers will be divided into halves for easy insertion and removal without necessitating the removal of the beam pipe.

Each chamber has a cell width along the beam of $2 \mathrm{~cm}$. The actual dritt chamber cell is separated by a fine mesh grid from a photon conversion gap which is $2.5 \mathrm{~cm}$. in width. A section of a chamber is shown in Fig. 11. The entire chamber volume is filled with a Xenon-Argon gas mixture to enhance the radiation length. The ionization clusters produced from TR photons in the conversion gap are slowly drifted through a uniform field region to the mesh grid. From there they are accelerated to one of the sense wires. The signals from the TR photon conversions are delayed in time by at least $100 \mathrm{nsec}$. from the primary charged particle signals, allowing them to be counted separately. The chamber closest to the interaction point will be used for charged particle tracking purposes only, but will be built using the same design as the others.

The construction of both the radiator blocks and the conversion gap drift chambers would take place at the Harvard High Energy Physics Lab. The facility which was used to construct the FEM system will be retooled for this task. This area consists of a large clean room and six large jig plate tables. A possible layout for the radiator block production is shown in Fig. 12. This facility will be available for this work starting Sept. 1986. It should be possible to complete the entire system in time for the second major CDF run in 1987.

Some tests are planned for early 1987 using up to five prototype transition radiator blocks and test chambers to determine the necessary parameters for the readout electronics. Figure 13 shows a cross section view of one of the test chambers. A small number of $100 \mathrm{MHz}$ flash ADC channels will be used for counting ionization clusters arriving from TR $X$-ray conversions in the conversion gap. The amplifier pulse shaping will be tuned in order to enhance the cluster counting efficiency. This study will be done by varying electron drift times in the conversion gap. The electric field in this gap can be varied independently on the test chamber. 


\section{Readout Electronics}

The readout electronics for this system must serve two purposes. First it must perform a normal drift chamber TDC function for tracking purposes. Secondly it must scale the number of hits in an extended time window in order to count TR photon conversions. The initial tracking function takes place in the first $=100$ nsec. after the beam crossing. The second scaling function extends over the next $\approx 2 \mu \mathrm{sec}$. It may be possible to implement a dynamic switching between these two functions, however, a simpler procedure is to split the signal and to perform the two operations in parallel.

The number of electronics channels per chamber is approximately 240 . When both ends are included this means of total of 2400 channels for the entire system.

\section{Cost Estimate}

Item

Cost

Radiator Block Materials

$\$ 35 \mathrm{~K}$

Drift Chamber Materials

Assembly Labor (2MY)*

$\$ 45 \mathrm{~K}$

Hardware and Supplies

$\$ 85 \mathrm{~K}$

Tooling

$\$ 30 \mathrm{~K}$

Shipping

Electronics (2400 channels)

$\$ 20 \mathrm{~K}$

$\$ 5 K$

\$230K

Total Cost

$\$ 450 \mathrm{~K}$

*This is additional labor - Harvard would supply approximately $4 \mathrm{MY}$ of technical labor to the project. 


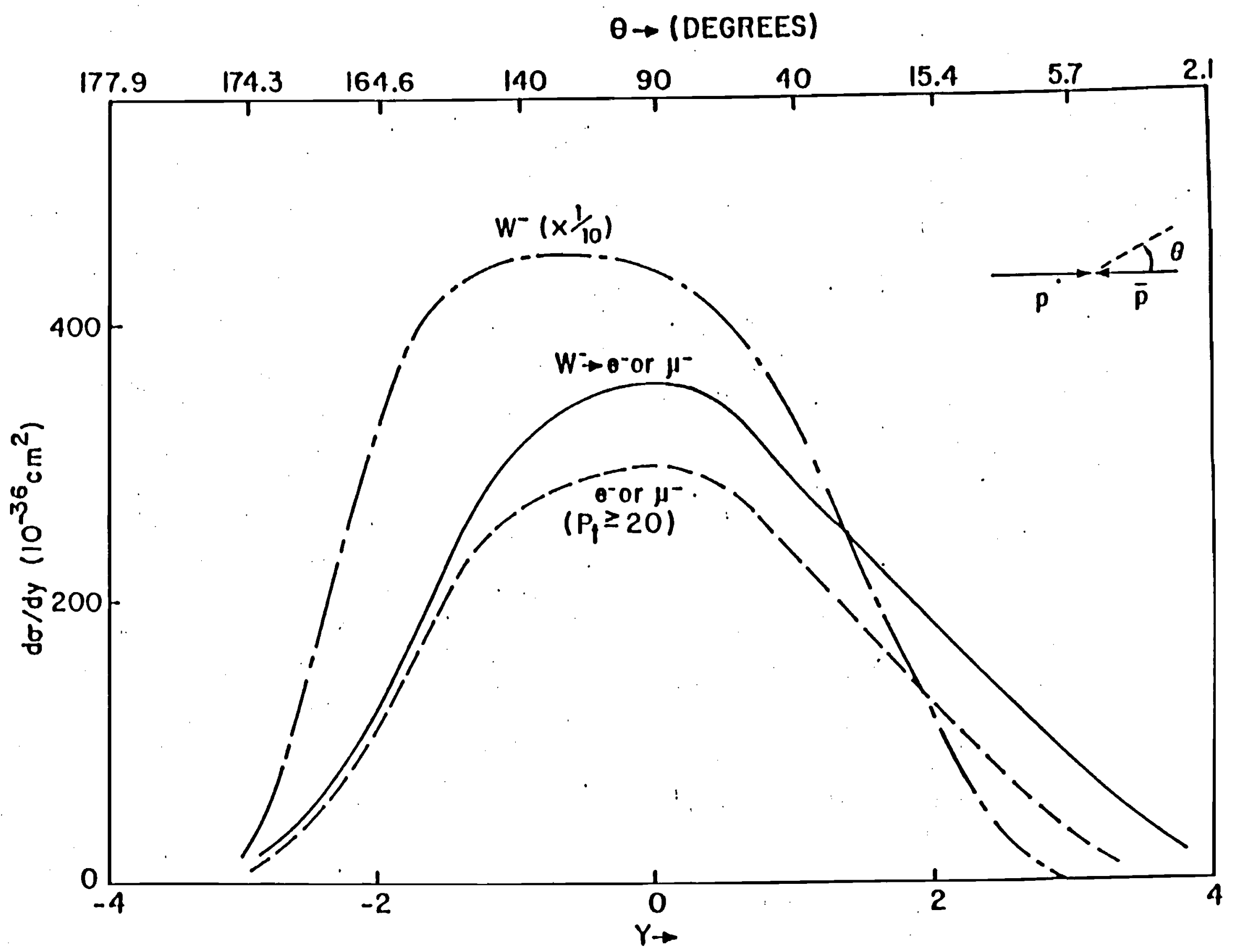

gure 1 


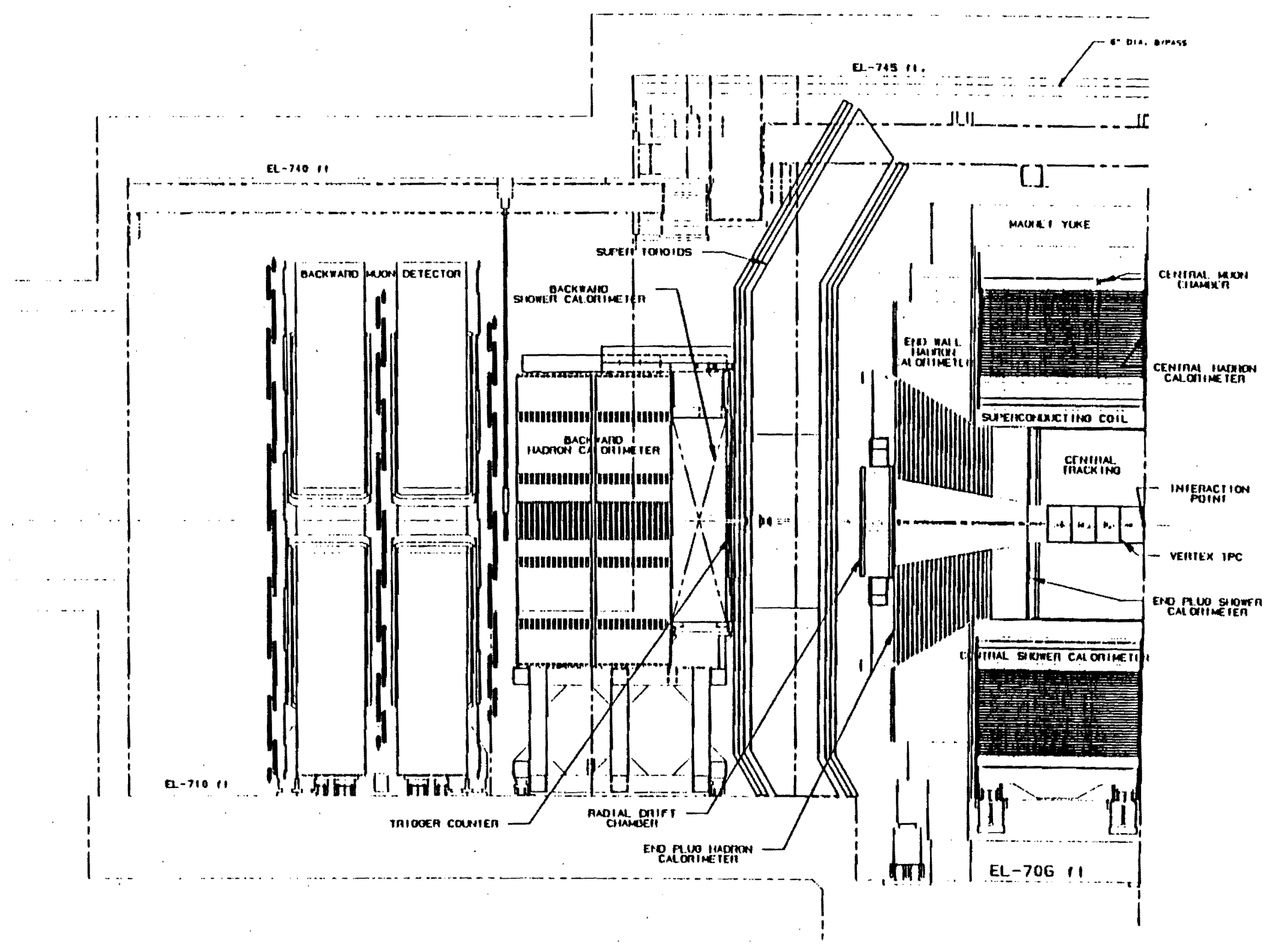

Figure 2 
FORWARD EM CALORIMETER, PLANE 1

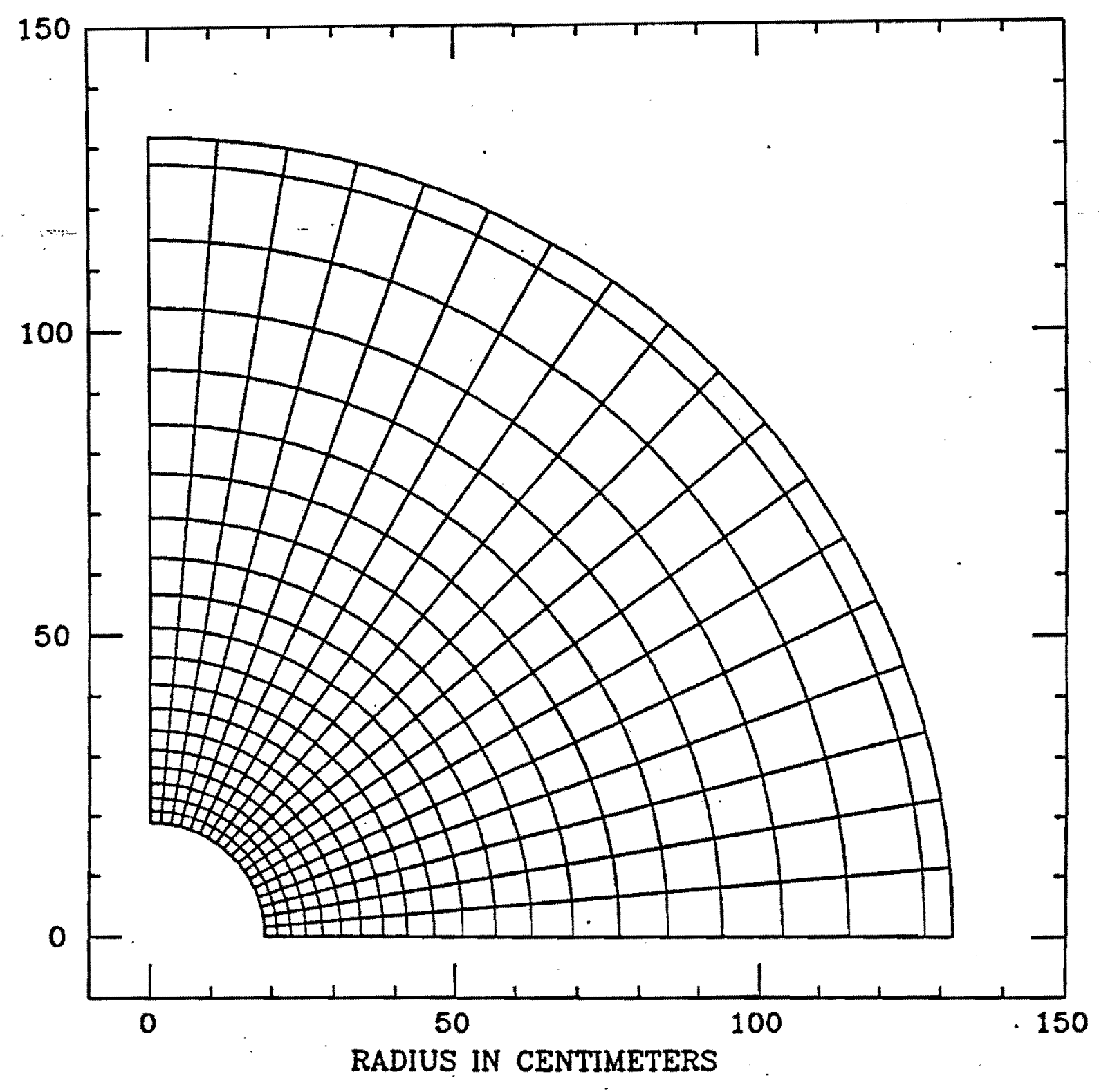

Figure 3 


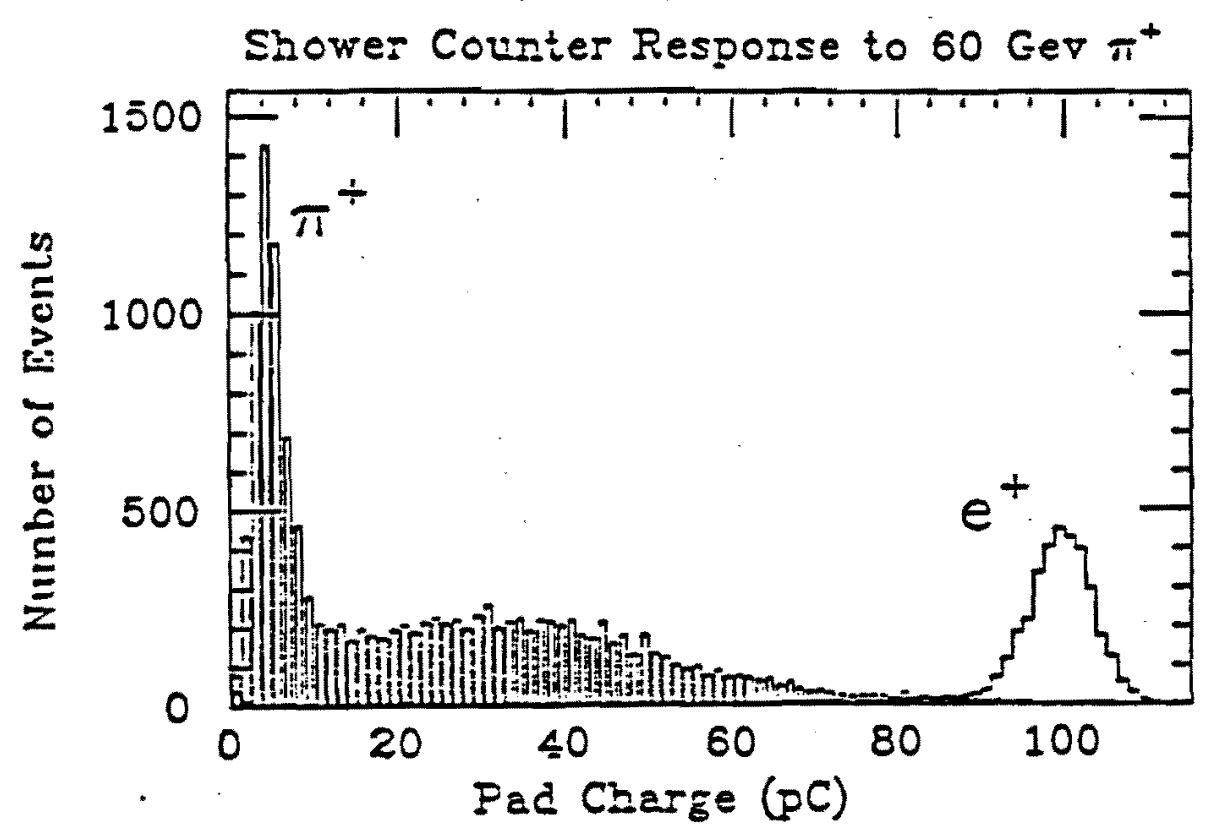

Figure 4 
Correlations in shower Counter Depth Segments at $60 \mathrm{Gev}$
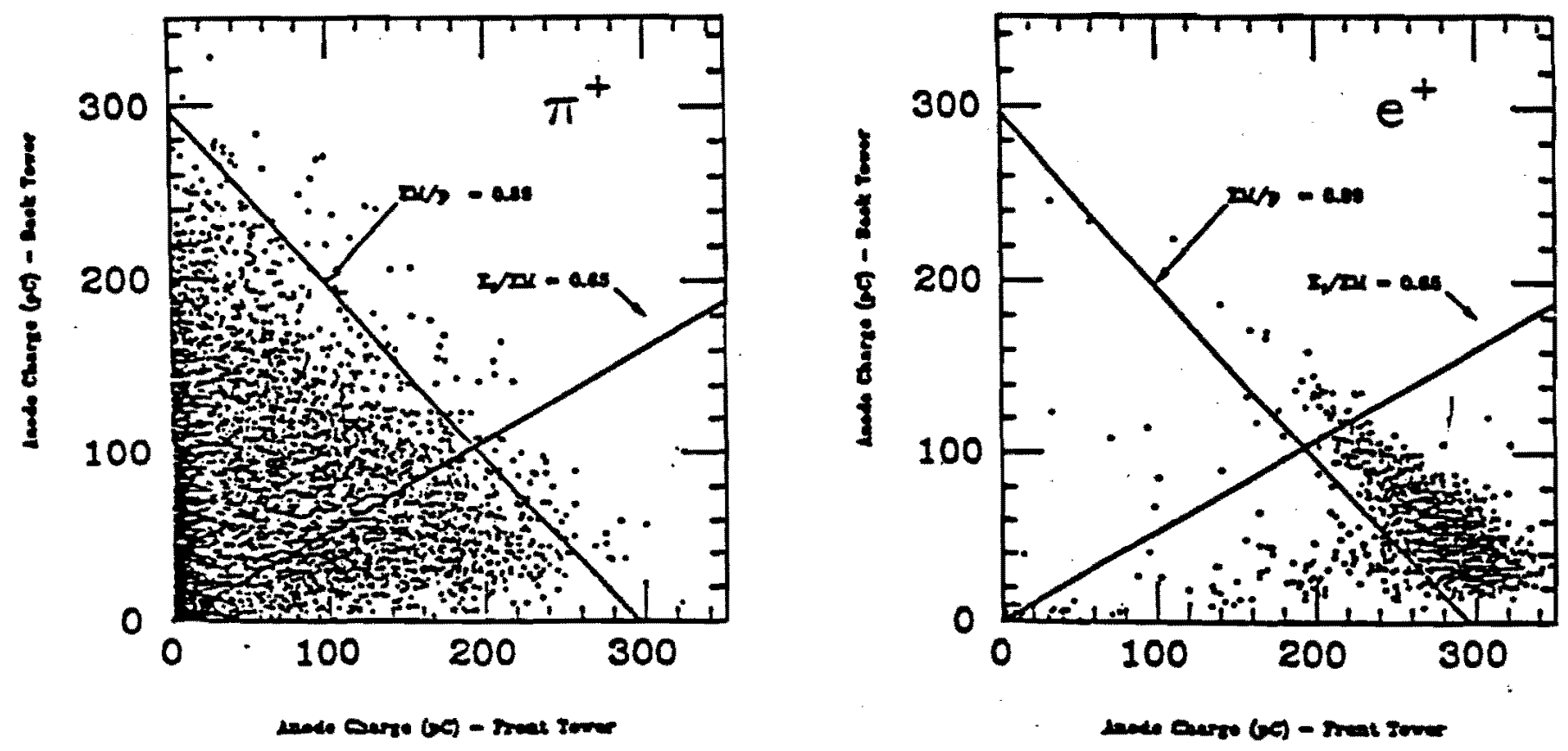

Figure 5 


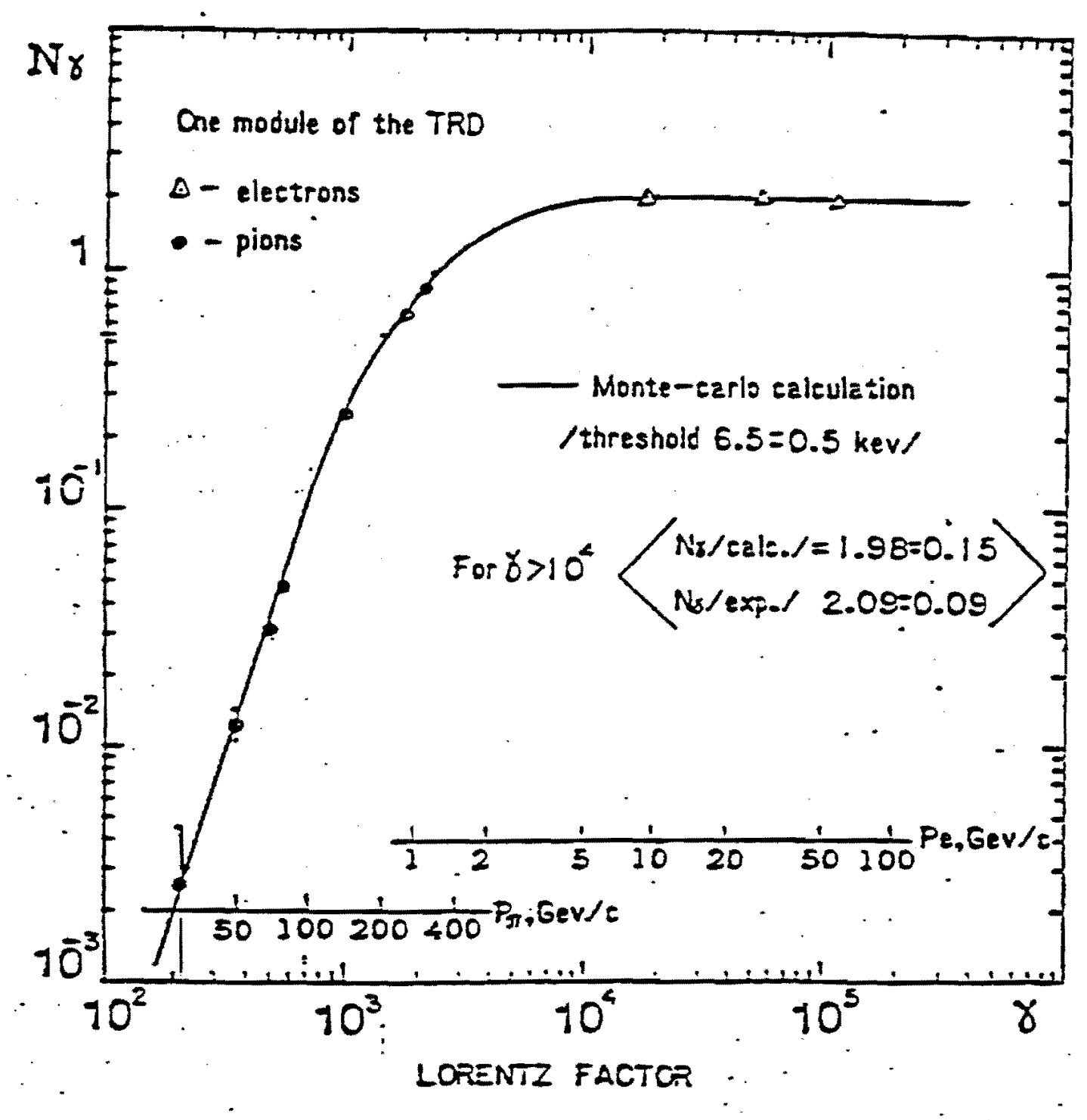

Figure 6 


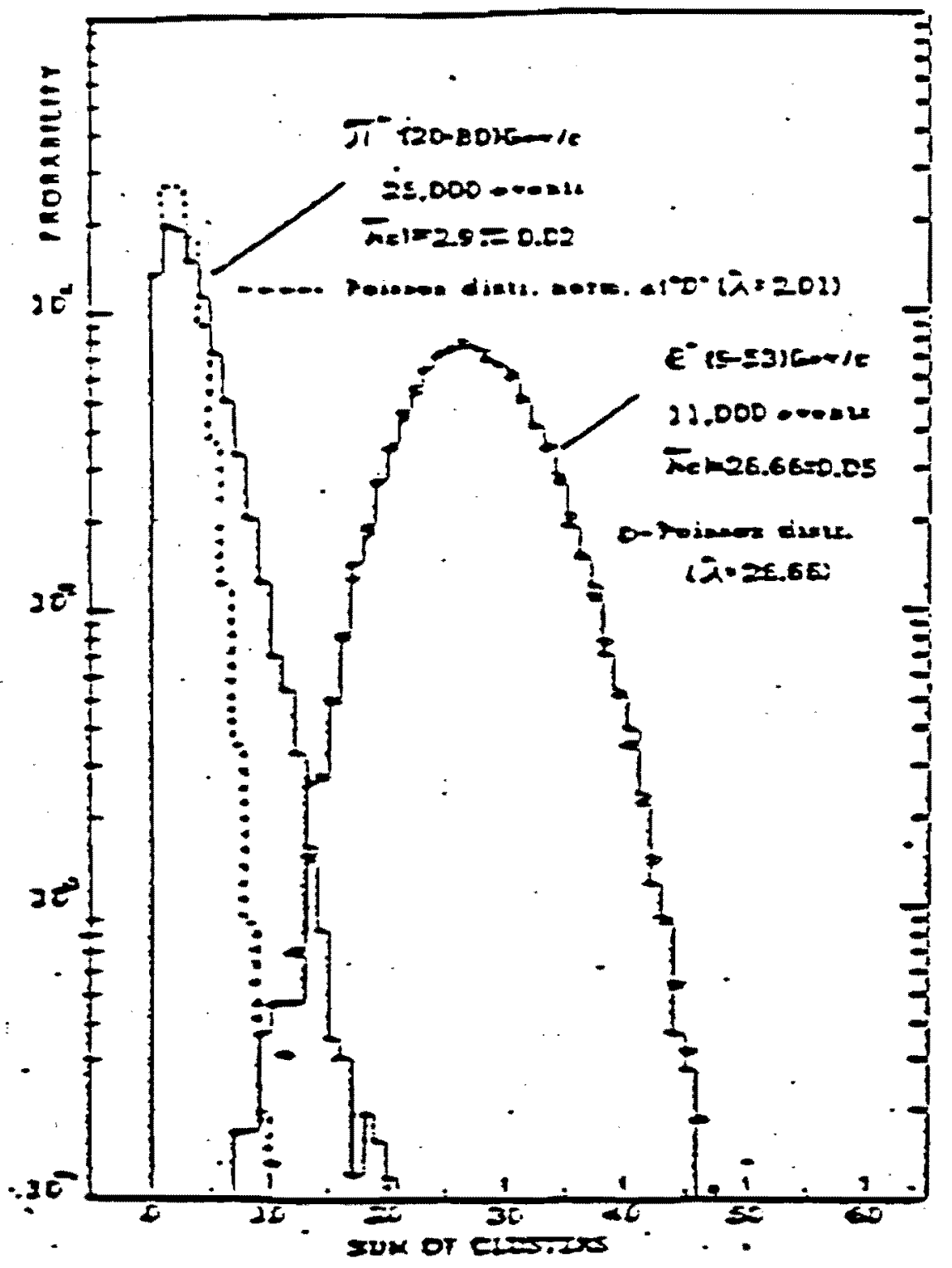

Figure 7 


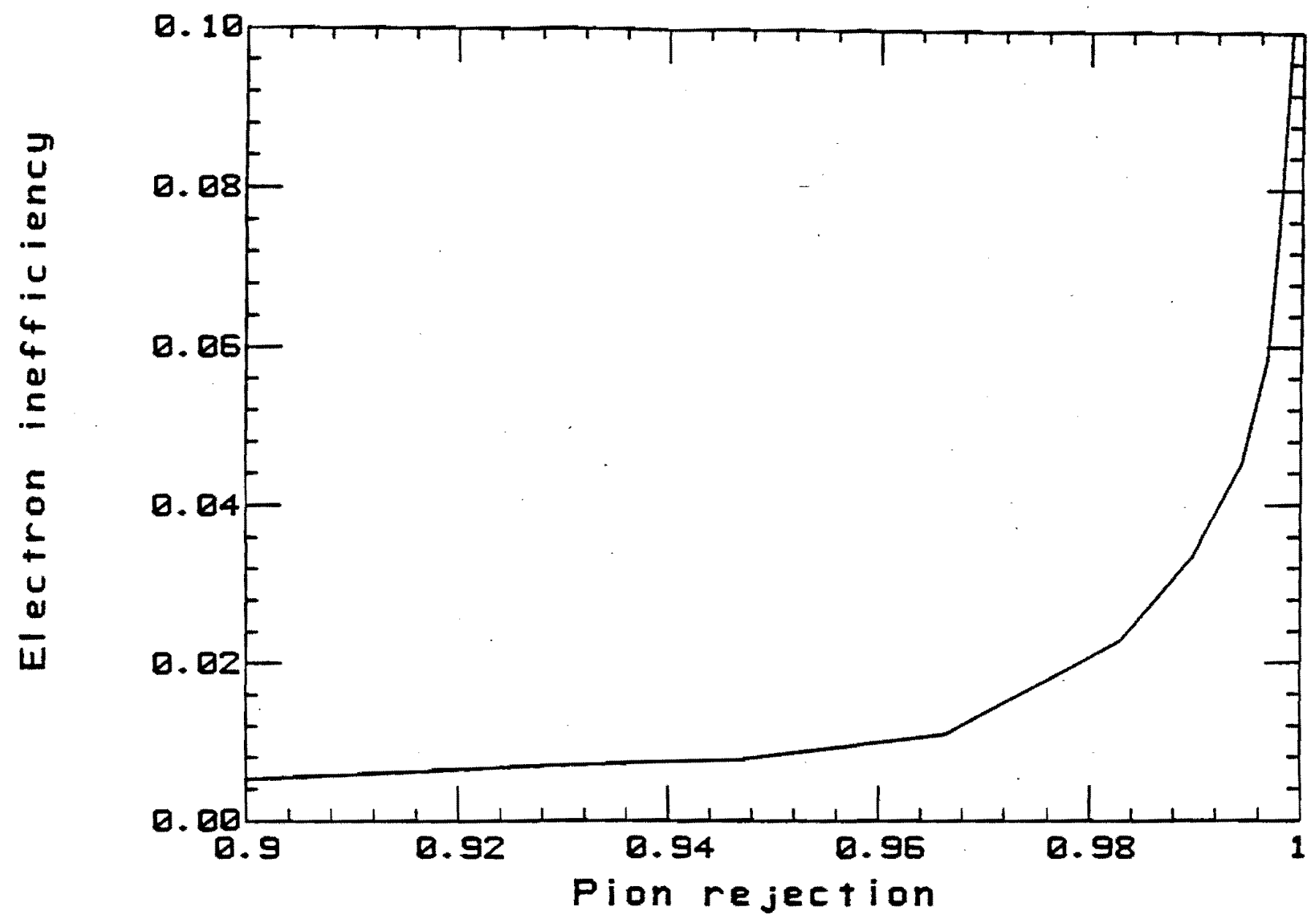

Figure 8 


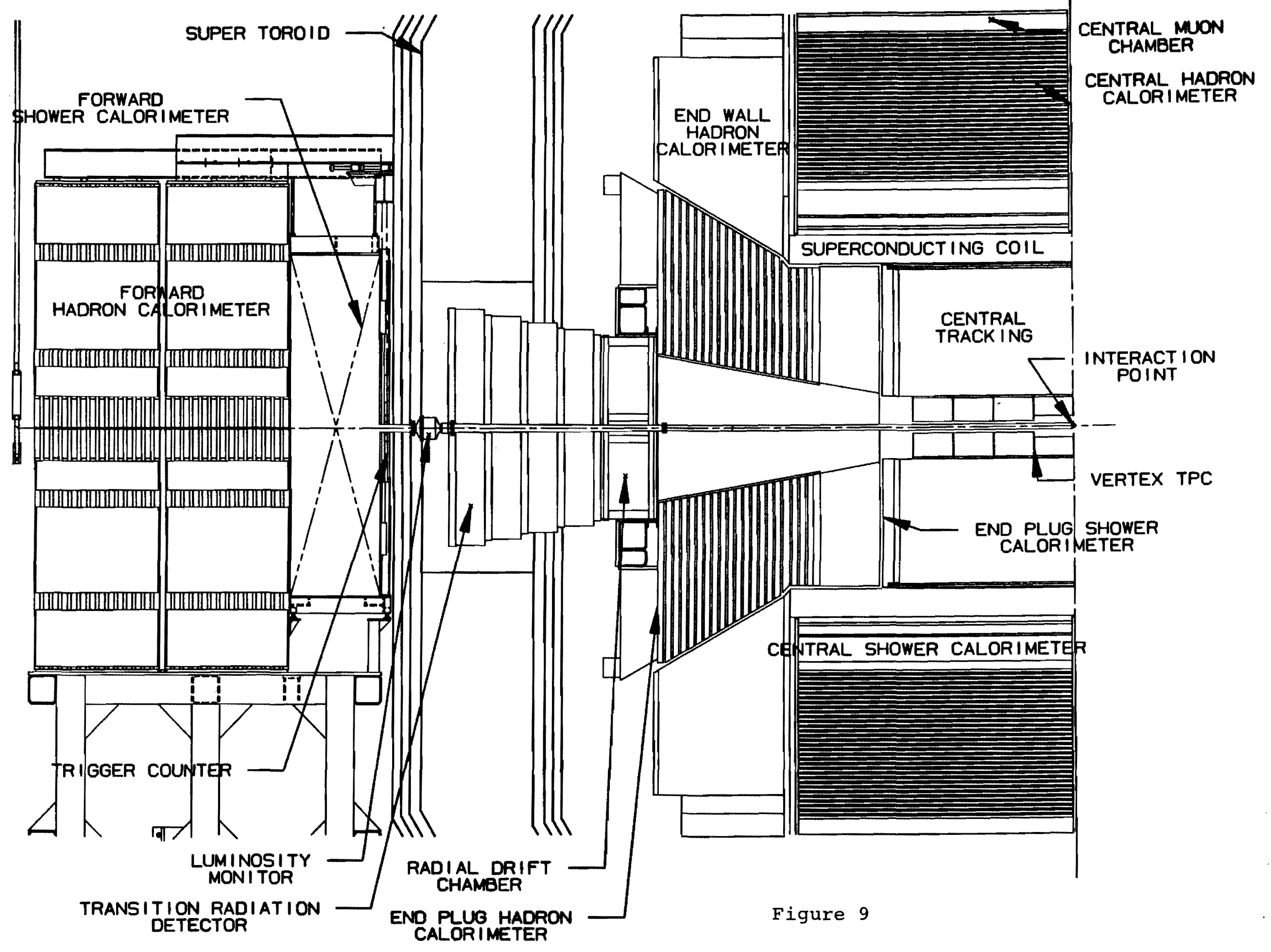




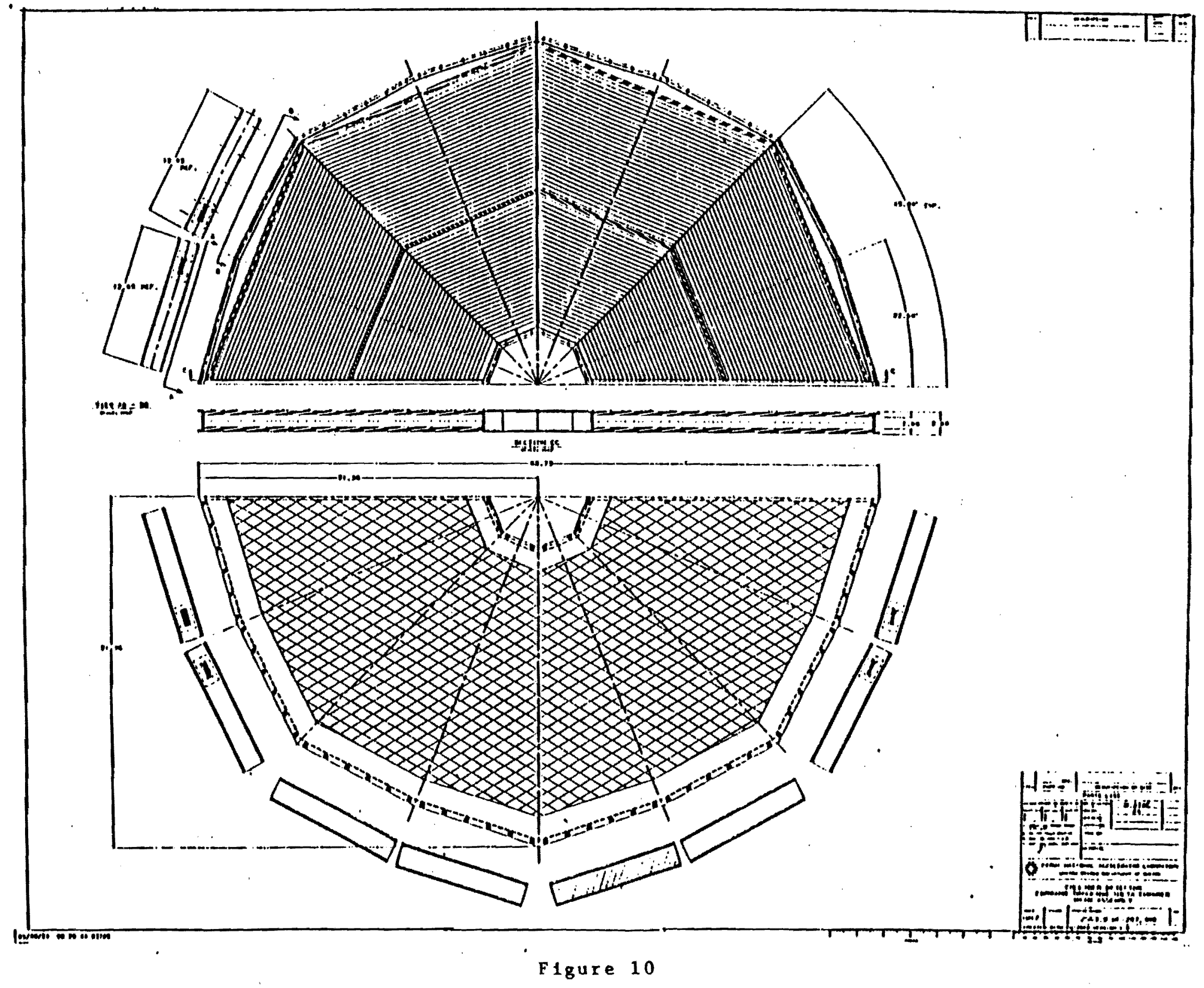




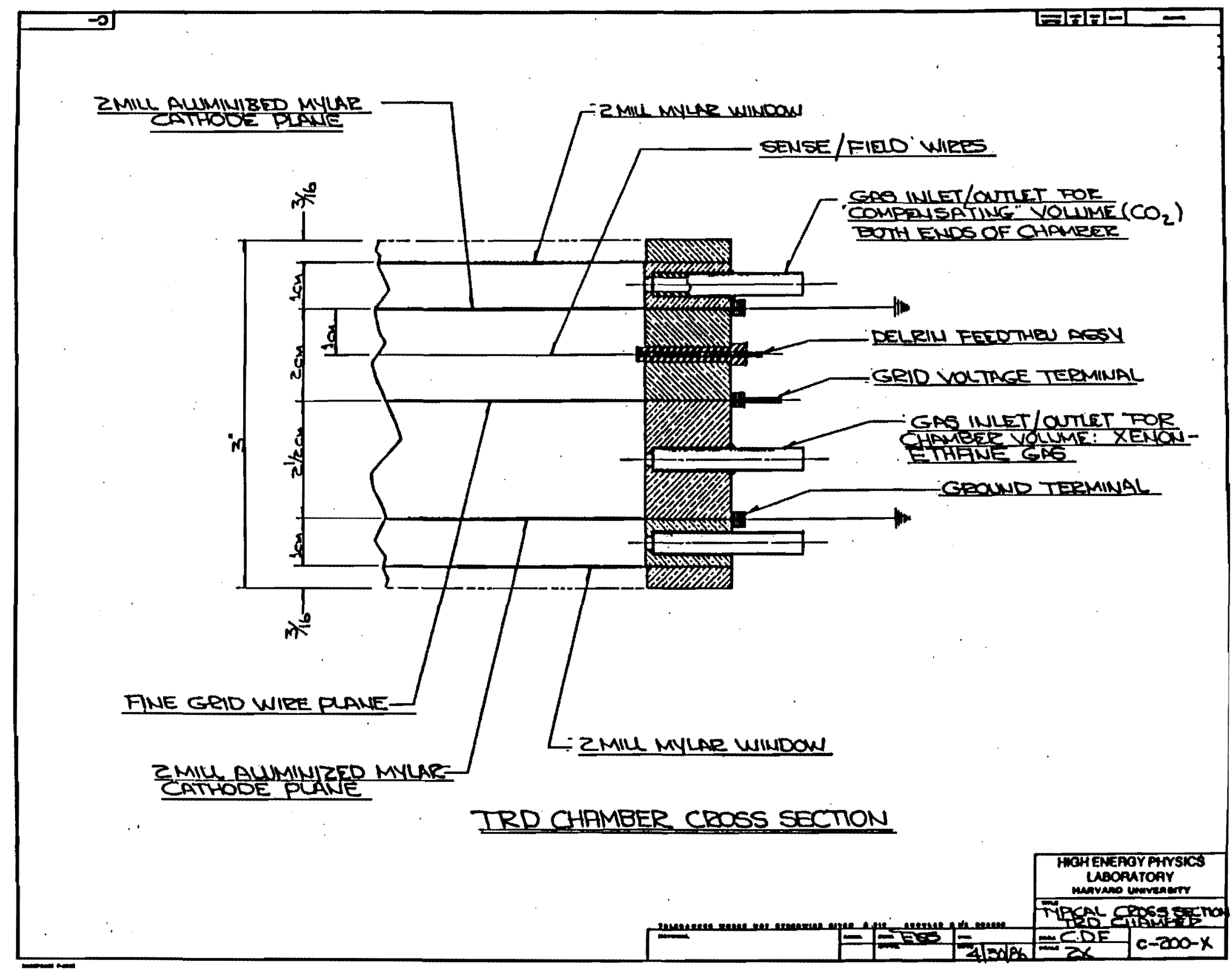

Figure 11 
Assintrey : ROON

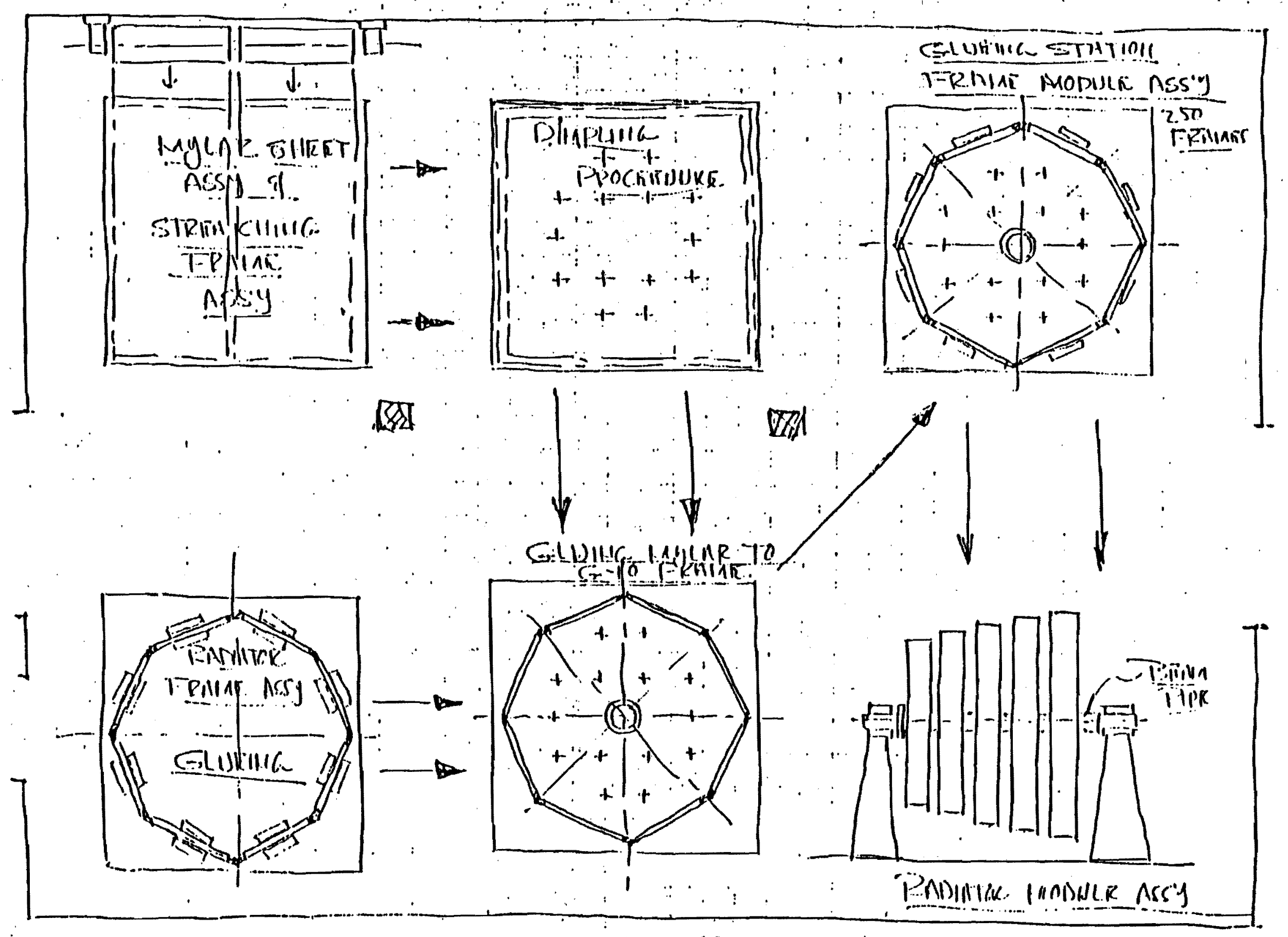

FIgure 12 


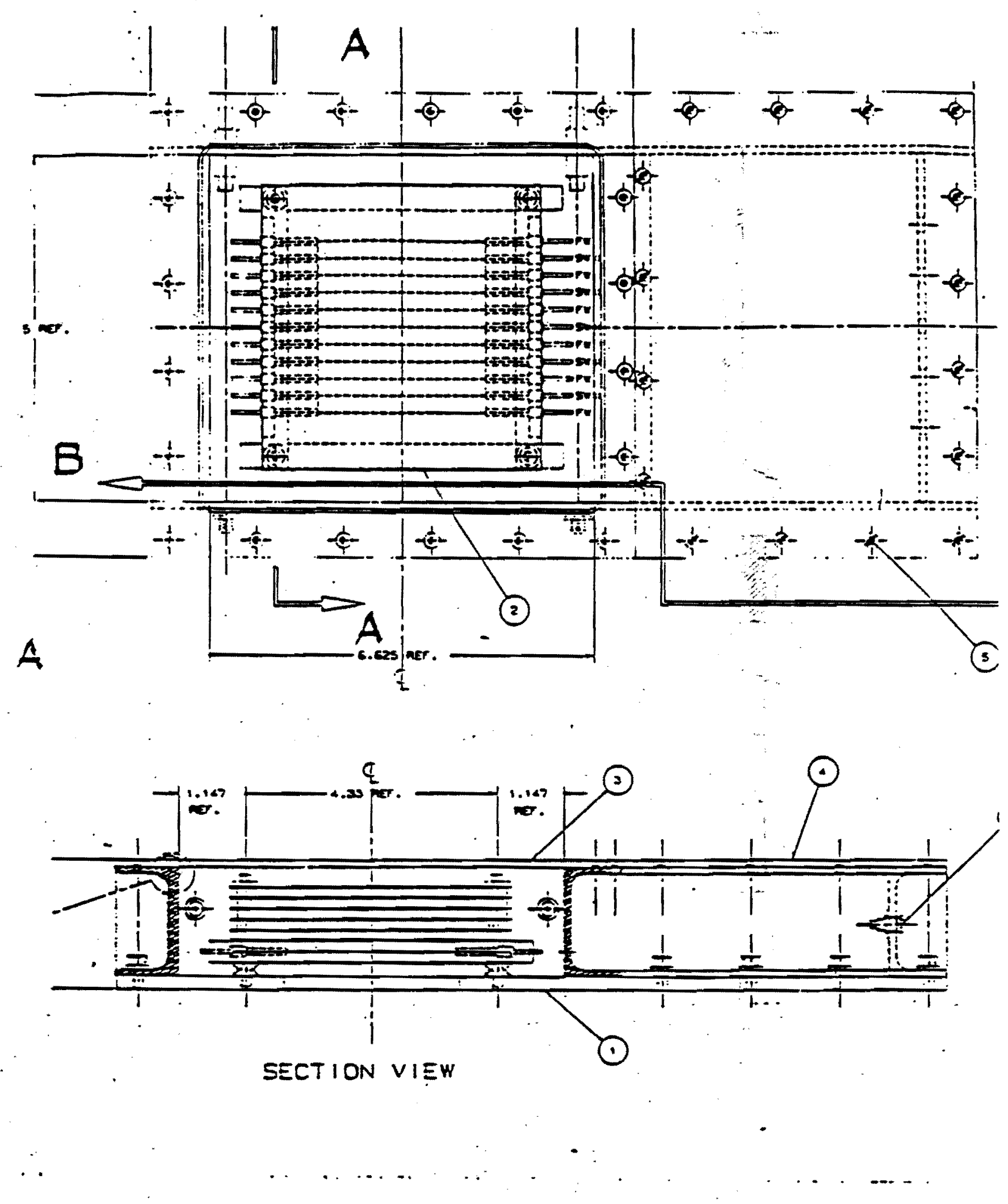

Figure i3 


\title{
Appendix E
}

\section{The Silicon Vertex (SVX) of CDF}

\author{
R. Kephart, A. Tol lestrup \\ Fermi National Accelerator Laboratory \\ W. Carithers, R. Ely, C. Haber \\ S. Kleinfelder, H. Spieler \\ Lawrence Berkeley Laboratory
}

F. Bedeschi, G. Bellettini, E. Focardi

A. Menzione, L. Ristori, G. Tonelli

INFN, Pisa, Italy

\section{Introduction}

The main task of $S V X$ is to provide a precise R-phi tracking of charged prongs as close as possible to the interaction point of CDF. This will allow the tagging of secondary vertices from decay of beauty and heavier flavours.

The layout consists of four layers of silicon crystals around the beam pipe as sketched in Fig. 1. The electrodes are thin longitudinal strips, parallel to the beam. The relevant parameters are listed in Table 1. The detector provides four points with a resolution of $15 \mu \mathrm{m}$ for each charged secondary between -2 $<y<2$, thus improving greatly the resolution in momentum and in impact parameter of CDF (see Table 2 for a comparison of CD resolutions with and without SVX).

In this crowded region of limited volume it is crucial to use a high granularity detector. In our proposed design, a total of 40,000 channels are separately amplified and multiplexed. This requires special electronics as will be discussed later.

Monte Carlo studies indicate that the system is CPBable of tagging secondary vertices with lifetimes between $10^{-13}=10^{-1}$
sec with an efficiency of the order of 30 percent. A primary goal is to tag the decay chain of heavy flavoured quarks. This will allow the detection of rare decay channels of new heavy particles involving $t, b$, c quark with reduced QCD background. The simulation studies show that the SVX can sort out signal from the background in cases of physical interest, and Table 3 quotes efficiencies for a few examples. 
The SVX places some undesirable material in the path of secondary particles in a delicate region upstream from the other detectors. Our design goal is a total weight of SVX of less than $1.5 \mathrm{Kg}$. The maximum thickness traversed by particles at any angle is $<2 \% x$ which reduces the number of secondary interactions to a tolefable level.

The readout system for the silicon strip detector poses many difficult problems. The pitch of the strip is $50 \mu$, and this must be matched to read out electronics and the channels multiplexed if one wishes to avoid 40,000 wires coming from the detector. This problem has been attacked in an elegant manner at Stanford by S. Parker and Terry Walker who have developed a multiplex chip which has all the electronics on it for reading out 128 channels in a serial manner. It can be "daisy chained" if desired and in addition has calibration circuits incorporated. However, there are several potential problems with this chip which are discussed below. As a result an alternate path is being pursued at LBL where a new chip is under development. At present it is not known which solution will be used. We describe here the two alternatives and indicate the approximate costs associated with each.

\section{IIa. Microplex Reädout Chip}

The present SVX design is based on the availability of a custom VLSI preamp matched to our strip geometry. The only presently existing chip which satisfies this constraint is the Microplex developed to read out the Mark II silicon vertex detector. The Microplex contains 128 channels on a $50 \mu \mathrm{m}$ pitch. Each channel has a charge sensitive preamp and two sample-and-hold capacitors for sampling the charge before and after beam crossing. With an on-chip shift register, the sampled charge can be serially multiplexed to a differential amplifier for external digitization. An early version of the Microplex gave a signal to noise ratio of 14 to 1 for minimum ionizing particles in a single crystal detector.

Although we are still considering this chip for our application, it still has some serious drawbacks. The first issue is power dissipation. The Microplex is implemented with nMOS technology and consequently generates about $20 \mathrm{~mW}$ per channel. Since the time between crossings at the Tevatron will be comparable to the settling time of the chip, the power cannot be turned off between crossings, and the device must be run DC. The heat of over 650 watts must be carried away by some cooling scheme that inevitably introduces unwanted material near the beam pipe. 
A second issue involves the effect of strip capacitance on the signal to noise ratio. Given the long interaction region at the Tevatron the vertex detector for CDF must have long strips and the source capacitance become correspondingly large. This increases the noise which depends I inearly on the source capacitance.

The third issue is readout speed. Again because of the short interbunch time, it is impossible at the Tevatron to read every channel on the Microplex between bunches. On the other hand this would be necessary if a secondary vertex trigger is to be developed.

These problems are all being studied. A test stand for the Microplex chip has been set up at LBL and reproduced at Fermilab and measurements on the electrical properties of the circuit are being made.

The problem of heat removal is under study at Pisa. Water cooling is very efficient but, of course, the additional material added to the system should be kept to a minimum. The present design of the end flanges of the detector is based upon $300 \mu \mathrm{m}$ thick Al pressed into a suitable shape as sketched in Fig. 2 . This provides the required mechanical strength, and heat exchange is provided by a 2,000 $\mu \mathrm{m} 0 . d$., $1600 \mu \mathrm{m}$ i.d. Al pipe in which water is circulated. The overall weight of an end plate is 120 gr.

Measurements on a prototype show that a water flow of 10 $1 /$ hour at a pressure of $1.5 \mathrm{Atm}$ can remove most of the heat with an increase of water temperature of $2-3 \mathrm{C}^{\circ}$. The prototype was also tested at a much higher pressure (6 Atm), and no difficulties were encountered, and the resulting flow was still linear.

At present, the most efficient dielectric material to be used as a substrate for the electronics is being studied. Alumina was used for the prototype tests. Boron nitride, Beryllium oxide, and other heat conductive ceramics are being considered. The construction schedule for the mechanical support calls for a first prototype in 1986.

II.b. LBL CMOS Extension of the Microplex

LBL is designing a CMOS enhancement of the Microplex to address the issues noted above for application at hadron colliders. The CMOS technology should reduce the power dissi- 
pation by about an order of magnitude. The signal-to-noise should also be significantly improved. A CMOS preamp should have a much higher single stage open loop gain which in turn results in a larger effective input capacitance for the preamp.

There are substantial architecture differences between the LBL design and the Microplex. The Microplex front end uses a conventional double sampling scheme to remove certain sources of noise. This scheme does not remove contributions of detector leakage current which can vary from channel to channel. The LBL design uses a novel switched capacitor circuit that is functionally equivalent to a triple sampling scheme that removes the leakage current contribution. In addition, a sparse data scan readout is being designed where every channel has a discriminator and latch. The reaidout speed should improve by the inverse of the occupancy - typically a factor of 10 to 100 .

A prototype design exists, and a preproduction prototype has been ordered for evaluation by July 1986. If this is successful, a production prototype could be ready by Dctober 1986.

\section{Costs}

The estimated costs of the vertex detector are shown below.

\section{SILICON VERTEX DETECTOR COSTS}

Equil $\frac{\text { Italy }}{\text { Eng }}$ Equip $\frac{\text { U.S. }}{\text { Eng }} \frac{\text { Eng }}{(M Y)}$

1. Silicon crystals

2. Preamp/Microplex 500 chips

3. Cables, electronics

4. Mechanical support, Tota 1
283

1

103

40

426
33

1

75

$1 / 2$

$1-1 / 2$
$1-1 / 2$

$3-1 / 2$ 


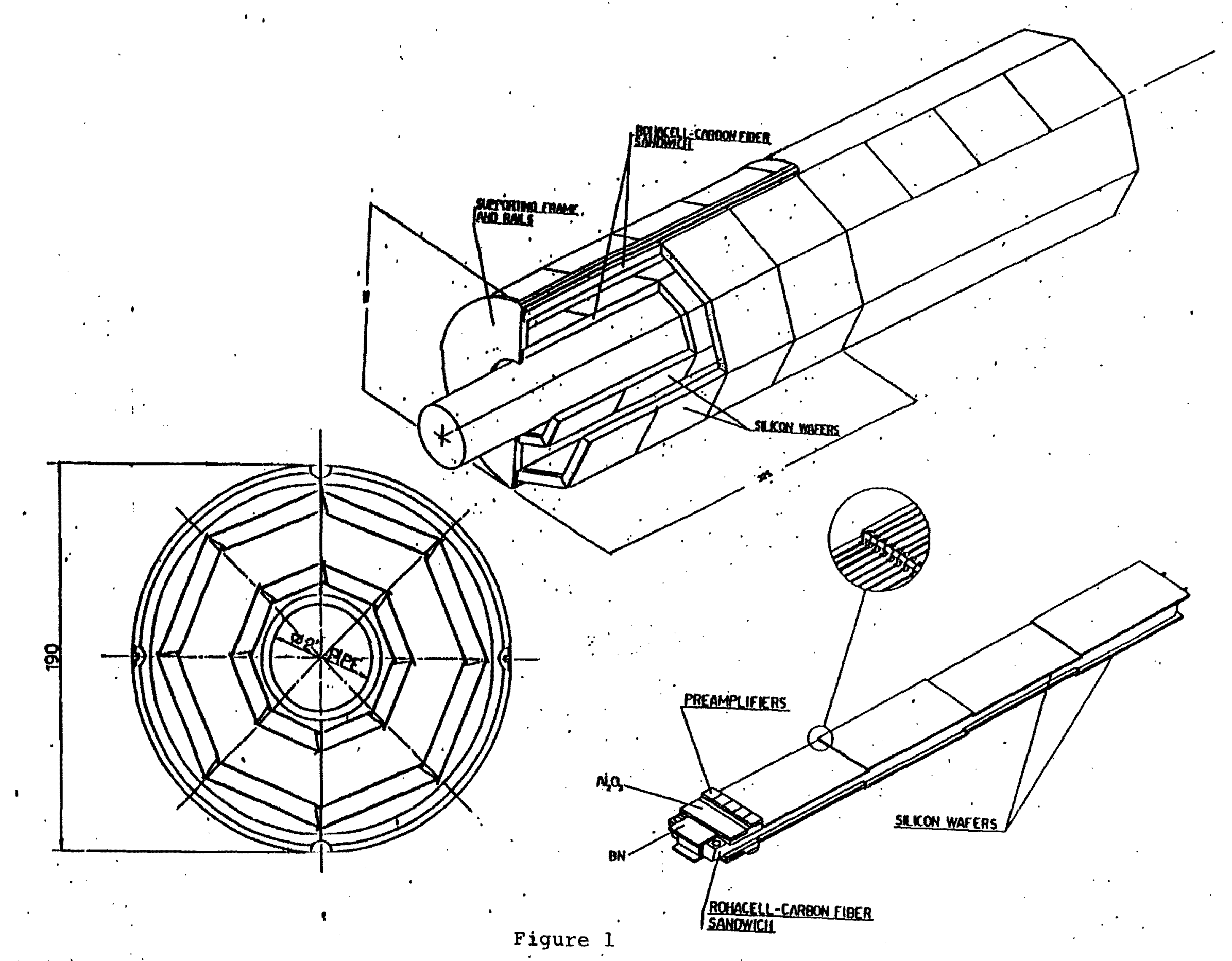

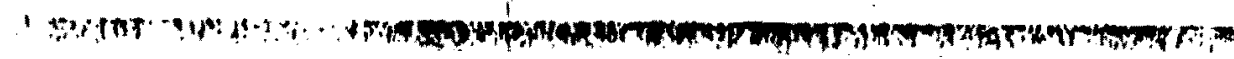

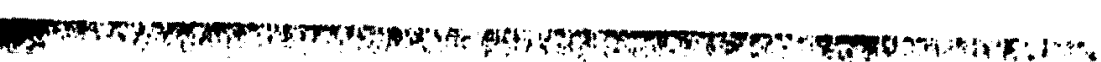


Table 1

\begin{tabular}{|c|c|c|c|c|c|c|c|}
\hline Layer & $\begin{array}{c}\text { Thickness } \\
\left(\mu_{m}\right)\end{array}$ & Shape & $\mid \begin{array}{l}\text { Internal } \\
\text { Diameter } \\
(\mathrm{mm})\end{array}$ & $\begin{array}{l}\text { Length } \\
(\mathrm{mm})\end{array}$ & $\begin{array}{c}\text { Size of } \\
\text { crystals } \\
\left(\mathrm{mm}^{2}\right)\end{array}$ & $\begin{array}{l}\text { Pitch } \\
(\mu \mathrm{m})\end{array}$ & Channels \\
\hline 1 & 200 & Octogonal & 30 & 600 & $26 \times 75$ & 50 & 8.032 \\
\hline 2 & 200 & $"$ & 43 & 600 & $33 \times 75$ & 50 & 11.200 \\
\hline 3 & 200 & $"$ & 70 & 600 & $48 \times 75$ & 100 & 7.680 \\
\hline 4 & 200 & $"$ & 80 & 600 & $55 \times 75$ & 100 & 8.800 \\
\hline
\end{tabular}

Table 2

\begin{tabular}{l|c|c|c} 
& $\begin{array}{c}\text { Momentum } \\
\text { Resolution } \\
\left(10^{-3} 6 \mathrm{e}^{-7}\right)\end{array}$ & $\begin{array}{c}\text { Polar } \\
\text { Resolution } \\
\text { (mad) }\end{array}$ & $\begin{array}{c}\text { Impact param. } \\
\text { Resolution } \\
(\mu \mathrm{mot})\end{array}$ \\
\hline without SVX & 1.0 & .9 & 200 \\
with SVX & .5 & .3 & 25 \\
& & &
\end{tabular}


Table 3

\begin{tabular}{l|r|r}
\multicolumn{1}{c}{ Process } & $\varepsilon_{3}(\%)$ & $\varepsilon_{4}(\%)$ \\
\hline$W \rightarrow t \bar{b}$ & 36.5 & 17.0 \\
$W \rightarrow c \bar{s}$ & 2.0 & 0.8 \\
$Q C D \rightarrow b \bar{b}$ & 35.3 & 22.4 \\
QCD $\rightarrow c \bar{c}$ & 1.8 & 0.4 \\
QCD $\rightarrow$. d.s.g. pairs & 0.8 & 0.4
\end{tabular}

SVX efficiency for tagging a number of physics channels. $\varepsilon_{3}=$ efficiency for finding a vertex containing three tracks. $\varepsilon_{4}=$ efficiency for finding a vertex containing four tracks. 


\section{APPENDIX F}

\section{COST OF CDF UPGRADE PROGRAY (FY868)}

$\begin{array}{lr}\text { SUPERTOROIDS } & \$ 3986 \mathrm{~K} \\ \text { TRD } & 450 \mathrm{~K} \\ \text { SILICON VERTEX } & 240 \mathrm{~K} \\ \text { LEVEL 3 TRIGGER } & \frac{367 \mathrm{~K}}{} \\ \text { CONTINGENCY (25\%) } & 1261 \mathrm{~K} \\ \text { SWF (15\%) } & 948 \mathrm{~K} \\ \end{array}$


CDF UPGRADE PROGRAY (FY 868)

\begin{tabular}{|c|c|c|c|c|}
\hline & FY87* & FY88 & FY89 & TOTAL \\
\hline SUPERTOROIDS & $\$ 333 K$ & \$1610K & $\$ 2043 \mathrm{~K}$ & $\$ 3986 \mathrm{~K}$ \\
\hline TRD & $250 \mathrm{~K}$ & $200 \mathrm{~K}$ & 0 & $450 \mathrm{~K}$ \\
\hline SIIICON VERTEX & $50 \mathrm{~K}$ & $190 \mathrm{~K}$ & 0 & 240K \\
\hline LEVEL 3 TRIGGER & $367 \mathrm{~K}$ & 0 & 0 & $367 \mathrm{~K}$ \\
\hline $\begin{array}{l}\text { FACILITY IMPROVEMENTS } \\
\text { (CONTINGENCY, SWF, MISC.) }\end{array}$ & $500 \mathrm{~K}$ & $1000 \mathrm{~K}$ & $957 \mathrm{~K}$ & $2457 \mathrm{~K}$ \\
\hline TOTALS & $\$ 1500 \mathrm{~K}$ & $\$ 3000 \mathrm{~K}$ & $\$ 3000 \mathrm{~K}$ & $\$ 7500 \mathrm{~K}$ \\
\hline
\end{tabular}

*note: FY87 is the final year of construction for CDF. Following the March, 1986 DOE/CDF review and additional FY88 funds, the current CDF budget plan calls for \$3.4M in FY87 equipment funds to complete the base detector. 